Revue d'histoire de l'Amérique française

REVUE D.HISTOIRE DE L'AMÉRIQUE FRANÇAISE

\title{
La disciplinarisation de l'histoire au Canada français, 1920-1950
}

\section{Patrice Régimbald}

Volume 51, numéro 2, automne 1997

Les pratiques de l'histoire de l'Amérique française depuis 50 ans

URI : https://id.erudit.org/iderudit/305645ar

DOI : https://doi.org/10.7202/305645ar

Aller au sommaire du numéro

Éditeur(s)

Institut d'histoire de l'Amérique française

ISSN

0035-2357 (imprimé)

1492-1383 (numérique)

Découvrir la revue

Citer cet article

Régimbald, P. (1997). La disciplinarisation de l'histoire au Canada français, 1920-1950. Revue d'histoire de l'Amérique française, 51(2), 163-200.

https://doi.org/10.7202/305645ar

\section{Résumé de l'article}

Cette étude est consacrée à l'examen de la construction d'une aire autonome de production et de transmisson du savoir historique au Canada français entre 1920 et 1950. Le faible développement des études historiques dans le système universitaire durant la période de l'entre-deux-guerres est en partie compensé par la multiplication, au même moment, des sociétés savantes qui s'affirment alors comme le lieu d'élaboration privilégié de l'historiographie canadienne-française. Ce milieu savant contribue à la mise en place graduelle d'un espace d'encadrement, de réunion, de production et de reconnaissance spécifique à l'histoire. Les fondations en 1947 des instituts d'histoire des universités Laval et de Montréal, de l'Institut d'histoire de l'Amérique française et de la Revue d'histoire de l'Amérique française se situent dans la continuité de ce mouvement, tout en posant les conditions institutionnelles propres à favoriser une prise de distance face aux différentes injonctions externes (doctrinales, idéologiques, politiques) qui structurent alors la quête de la vérité et l'étude du passé.
Ce document est protégé par la loi sur le droit d'auteur. L'utilisation des services d'Érudit (y compris la reproduction) est assujettie à sa politique d'utilisation que vous pouvez consulter en ligne.

https://apropos.erudit.org/fr/usagers/politique-dutilisation/ 


\title{
LA DISCIPLINARISATION DE L'HISTOIRE AU CANADA FRANÇAIS 1920-1950
}

\author{
PATRICE RÉGIMBALD \\ Département d' histoire \\ Université du Québec à Montréal
}

\section{RÉSUMÉ}

Cette étude est consacrée à l'examen de la construction d'une aire autonome de production et de transmisson du savoir historique au Canada français entre 1920 et 1950. Le faible développement des études historiques dans le système universitaire durant la période de l'entre-deux-guerres est en partie compensé par la multiplication, au même moment, des sociétés savantes qui s'affirment alors comme le lieu d'élaboration privilégié de l'historiographie canadienne-française. Ce milieu savant contribue à la mise en place graduelle d'un espace d'encadrement, de réunion, de production et de reconnaissance spécifique à l'histoire. Les fondations en 1947 des instituts d'histoire des universités Laval et de Montréal, de l'Institut d'histoire de l'Amérique française et de la Revue d'histoire de l'Amérique française se situent dans la continuité de ce mouvement, tout en posant les conditions institutionnelles propres à favoriser une prise de distance face aux différentes injonctions externes (doctrinales, idéologiques, politiques) qui structurent alors la quête de la vérité et l'étude du passé.

\section{ABSTRACT}

This paper analyses the process of formation of a relatively autonomous space of production and diffusion of historical knowledge in French Canada between 1920 and 1950. Between the two World Wars, the teaching of history was marginal in the university curriculum and the elaboration of a French Canadian historiography found its way through local historical societies, which gradually provided sites for meetings, discussion and publication as well as intellectual and social recognition. The creation in 1947 of the Instituts d'histoire at Université Laval and Université de Montréal, as well as the foundation of the Institut d'histoire de l'Amérique française and the Revue d'histoire de l'Amérique française, must be seen in light of these previous activities, though they also fix the institutional conditions for a practice of history less directly submitted to external factors, be they political, doctrinal, or ideological. 
Il est un lieu commun que d'associer la période de l'immédiat après-guerre à un tournant important dans l'évolution de la discipline historique au Canada français. En l'espace de quelques mois, fin de 1946 et début de 1947, quatre institutions majeures sont créées: l'Institut d'histoire de l'Université de Montréal, l'Institut d'histoire et de géographie de l'Université Laval et l'Institut d'histoire de l'Amérique française qui, à peine fondé, lance la Revue d'histoire de l'Amérique française. La génération d'historiens universitaires qui, les premiers, ont animé et assuré la réussite de ces institutions, ont propagé l'idée qu'une coupure profonde, définitive, se serait alors établie entre deux états successifs de la pratique historienne, le premier, antérieur à ces fondations, étant marqué du sceau de l'amateurisme, le second, inaugurant l'ère nouvelle de l'histoire professionnelle'. En somme, 1947 serait l'année zéro de la professionnalisation de l'histoire au Canada français. Les anniversaires, comme en cette année de demi-siècle, occasions tantôt de célébrations, tantôt de retours critiques sur le passé, tendent à accréditer cette idée d'une naissance, située et datée précisément au moment de la mise au monde, sans que ne soit examinée la période plus ou moins longue de gestation qui l'a précédée.

Notre propos, dans cette étude, est d'éclairer la genèse de ce «big bang historiographique» en nous penchant sur le «moment d'avant». L'examen de la période longue (1920-1950) dans laquelle s'inscrivent ces fondations permettra de dresser un état des lieux des institutions historiennes canadiennes-françaises et de montrer qu'un espace de production et de reconnaissance propre à l'histoire commence à se constituer dès les années 1920.

Une précision s'impose sur la notion de professionnalisation qui a souvent été utilisée par les historiens pour décrire tout un ensemble de transformations survenues dans l'évolution de la discipline historique au $\mathrm{XX}^{\mathrm{e}}$ siècle: adoption des règles de la critique historique, revendication d'objectivité, abandon des formes narratives traditionnelles, développement d'un enseignement spécialisé et d'une aire autonome de recherche en milieu universitaire, formation d'un nouvel ethos professionnel fondé sur le contact direct avec les sources, élévation d'une activité pratiquée en dilettante à une occupation dont on peut

1. Voir «Un combat pour la nouvelle histoire», dans Marcel Trudel, Mémoires d'un autre siècle (Montréal, Boréal, 1987), 167-206; "Histoire et historiens", dans Michel Brunet, Canadians et Canadiens. Études sur l'histoire et la pensée des deux Canadas (Montréal, Fides, 1954), 33-46; Guy Frégault, «Histoire, traditions et méthodes», Action universitaire, 14,1 (octobre 1947): 35-42; Guy Frégault, «Antiquaires et historiens», Notre temps (22 août 1953): 1. 
tirer ses moyens d'existence, etc. ${ }^{2}$. À la suite d'Yves Gingras ${ }^{3}$, nous proposons de limiter l'usage du terme de professionnalisation à la description du processus de maîtrise d'une occupation par un groupe, la profession ne constituant qu'une forme particulière de cette mâ̂trise. Or la profession entendue comme sous-ensemble d'activités les professions libérales - disposant de l'autorité légale de régler l'accès au titre et aux postes nous apparaît peu appropriée pour décrire l'organisation de l'institution historienne ${ }^{4}$. Nous préférons la notion de discipline qui est associée, dans les travaux des sociologues des sciences, à la forme organisationnelle privilégiée dans les processus historiques de différenciation des sciences et d'institutionnalisation sociétale de la recherche ${ }^{5}$. Elle définit un espace distinct de production et de transmission du savoir et un espace d'évaluation et de reconnaissance autonome, qui fonctionne selon ses propres règles et où domine le jugement des pairs comme premier principe de hiérarchisation. La notion de disciplinarisation renvoie quant à elle au processus de la mise en place de cet espace institutionnel, dont la spécification et l'autonomie constituent les enjeux principaux. Parvenue à une certaine stabilité, la forme disciplinaire est caractérisée par la lutte entre les agents, à l'intérieur même de la discipline constituée, pour l'obtention

2. Parmi les auteurs qui ont étudié le développement de l'histoire en termes de professionnalisation, mentionnons pour l'Angleterre: John Kenyon, The Historical Profession in England Since the Renaissance (London, Weidenfeld and Nicolson, 1983), 322 p.; Philippa Levine, The Amateur and the Professionnal. Antiquarians, Historians and Archeologists in Victorian England (Cambridge, Cambridge University Press, 1986), 210 p.; Doris S. Goldstein, «The Professionnalization of History in the Late Nineteenth Century and Early Twentieth Century», Histoire de l'historiographie, 3 (1983): 3-27. Pour la France: William Keylor, Academy and Community. The Foundation of French Historical Profession (Cambridge, Harvard University Press, 1975), 286 p.; Olivier Dumoulin, Profession historien (1919-1939). Un métier en crise, Paris, thèse de l'École des hautes études en sciences sociales, 1983), 429 p.; Christophe Charle, «Être historien en France: une nouvelle profession», dans François Bédarida, $L^{\prime}$ histoire et le métier d'historien en France 1945-1995 (Paris, Éditions de la Maison des sciences de l'homme, 1995), 21-44. Pour les États-Unis: John Highham, Leonard Krieger et Felix Gilbert, History. Professionnal Scholarship in America (New York, Garlang Publishing, 1985), 402 p.

3. Yves Gingras, «L'institutionnalisation de la recherche en milieu universitaire et ses effets», Sociologie et sociétés, 23,1 (printemps 1991): 42-43.

4. Le pouvoir de maitrise de la discipline, dans le cas de l'histoire, ne s'exerce tout au plus que sur les recrues, par la sanction des compétences à travers l'enseignement spécialisé. Mais aucun mécanisme établi ne permet de décréter qui est historien et qui ne l'est pas. De plus, dans sa logique de fonctionnement comme système d'interrelations et d'échanges, la discipline s'apparente à un marché à consommation restreinte - fermé selon Gingras - où les producteurs de savoir sont souvent les seuls consommateurs alors que «la profession est plutôt caractérisée par le fait que les agents circulent dans un marché ouvert où les clients ne sont pas eux-mêmes producteurs de savoir». Yves Gingras, op. cit., 43.

5. Voir, par exemple, G. Lemaine et al., Perspectives on the Emergence of Scientific Disciplines (Chicago, Aldine, 1976), 281 p.; Rudolph Stichweh, Étude sur la genèse du système scientifique moderne (Lille, Presses de l'Université de Lille, 1991), 209 p. 
du pouvoir de définir les objets légitimes de la recherche, le corpus disciplinaire à être transmis, le curriculum, les règles d'apprentissage, etc. ${ }^{6}$. Mais avant cela, encore faut-il qu'une aire de la recherche ait été délimitée et qu'à l'intérieur du champ scientifique, intellectuel et universitaire, un groupe ait réussi à obtenir le pouvoir d'exercer une gestion collégiale sur la production et la reproduction des connaissances.

Nous pensons qu'un tel espace de production et de reconnaissance, dans le domaine de l'histoire, a commencé à se mettre en place durant les années 1920 au Canada français. Cependant, une des particularités de sa naissance et de son évolution réside dans le fait que le processus de spécification s'est d'abord effectué hors de l'université - où s'active habituellement ce mécanisme de différenciation intellectuelle - pour prendre plutôt place dans le cadre de sociétés savantes. Ce n'est que par la suite, et en partie sous l'impulsion de ces regroupements savants, que le mouvement atteindra l'université.

Nous chercherons, dans la première partie, à montrer la place marginale de l'histoire à l'intérieur du système universitaire avant 1945 et à illustrer sa domination pour ainsi dire structurale par la littérature et la philosophie. Puis nous nous pencherons sur le regroupement parallèle de nombreux historiens, à l'intérieur de sociétés savantes, qui permet une première forme de gestion collégiale de la production historiographique, mais où les enjeux sociaux, politiques et idéologiques continuent de structurer l'écriture de l'histoire. Enfin, nous examinerons les fondations de 1947 en cherchant à les réinsérer dans leur contexte et à pondérer leur contribution dans la constitution d'une discipline autonome.

Une dernière remarque s'impose sur la délimitation de cet espace aux seules limites du Canada français. Ce découpage pourrait sembler arbitraire si l'évolution différenciée et distincte de deux traditions historiographiques, l'une de langue française, l'autre de langue anglaise, inscrites dans les institutions par les acteurs eux-mêmes, n'imposait toute la force de son évidence à l'observateur. Disons seulement que l'illustration parfois insistante de cette dualité institutionnelle ne constitue qu'un aspect collatéral de la démonstration principale.

6. Michel Leclerc, «La notion de discipline scientifique et ses enjeux sociaux», Politique, 15 (1989): 23-51; Alberto Cambrosio et Peter Keating, «The Disciplinary Stake: the Case of Chronobiology», Social Studies of Science, 14 (1983). 


\section{L'HISTOIRE DANS LE SYSTÈME UNIVERSITAIRE AU CANADA FRANÇAIS AVANT 1945: INDIGENCE ET SUBORDINATION}

La phase industrielle du début du $\mathrm{XX}^{\mathrm{e}}$ siècle, centrée sur les nouvelles technologies de l'hydro-électricité et des processus chimiques, provoque une remise en question du système d'éducation au Canada français. C'est que les nouveaux besoins en personnel hautement qualifié ne sont que très imparfaitement comblés par la main-d'œuvre locale. Certains analystes, tel Errol Bouchette, s'inquiètent de l'indépendance économique des Canadiens français et proposent un programme de reconquête économique centré sur le développement de l'enseignement technique et professionnel. Le gouvernement de Lomer Gouin, sensible à ces appels, crée une école d'arpentage affiliée à l'Université Laval en 1907, y ajoute une école de foresterie en 1910, tandis que l'École des hautes études commerciales ouvre ses portes à Montréal la même année. L'organisation de l'université autour des quatre facultés traditionnelles - théologie, droit, médecine et arts - apparaît mal adaptée aux conditions nouvelles. L'autonomie conquise par la succursale montréalaise de l'Université Laval en 1919 donne le coup d'envoi à un réaménagement des structures. L'Université de Montréal crée trois nouvelles facultés en 1920-1921 - lettres, sciences et philosophie - ainsi qu'une École des sciences sociales. L'Université Laval, qui ne veut pas être en reste, met sur pied l'École normale supérieure - destinée à la formation des maîtres en lettres et en sciences - et l'École supérieure de chimie. Cette période effervescente, dite de «fondations» par les historiens des sciences, est caractérisée par la création de nombreuses institutions de haut savoir et par la lutte pour l'acquisition d'une légitimité culturelle pour l'activité scientifique ${ }^{7}$.

C'est dans ce contexte que l'histoire fait son apparition à l'université. Mais son développement comme discipline distincte met du temps à se réaliser. Alors qu'au début des années 1940, les universités canadiennes-anglaises peuvent s'enorgueillir d'un effectif de près de 400 étudiants réguliers en histoire, de trois programmes de doctorats et d'une dizaine à la maîtrise ${ }^{8}$, la discipline historique n'occupe aucune place spécifique dans l'université au Canada français, ni

7. Marcel Fournier, «Science culture et société au Québec», L'entrée dans la modernité. Science, culture et société au Québec (Montréal, Albert Saint-Martin, 1986), 13-40. Voir également Raymond Duchesne, La science et le pouvoir au Québec (1920-1965) (Québec, Éditeur officiel du Québec, 1978), 126 p.; Luc Chartrand, Raymond Duchesne et Yves Gingras, Histoire des sciences au Québec (Montréal, Boréal, 1987), 487 p.

8. Archange Godbout, «L'histoire dans nos universités», Culture, 2,1 (mars 1941): 4150. Voir également Reginald G. Trotter, «Historical Research in Canada», Canadian Historical Review, 20,3 (septembre 1939): 251-257. 
comme enseignement spécialisé ni comme aire autonome de recherches. Lionel Groulx notait déjà en 1929, dans un mémoire pour le développement des études d'histoire canadienne présenté à la Faculté des lettres de l'Université de Montréal, «l'indigence de notre production historique comparée à celle des anglos-canadiens ${ }^{9} »$. Parmi les causes de cette indigence, Groulx identifiait «le peu de stimulants accordés par nos universités de langue française aux études d'histoire du Canada [et] une insuffisante orientation des étudiants vers le choix de thèses d'histoire canadienne pour la conquête de diplômes universitaires ${ }^{10}$ ».

Quelques chaires d'histoire sont distribuées ici et là dans les différentes facultés universitaires: chaires d'histoire ecclésiastique à la Faculté de théologie de l'Université d'Ottawa occupées par les oblats Georges Simard (1916-1947) et Edgard Thivierge; chaires d'histoire du Canada à la Faculté de droit de l'Université Laval et à la Faculté des lettres de l'Université de Montréal détenues respectivement par Thomas Chapais de 1919 à 1934 et Lionel Groulx de 1915 à 1948. À la Faculté des arts de l'Université Laval, deux expériences demeurent sans lendemain: un cours d'histoire des Amériques par l'abbé Arthur Maheux en 1921 et un cours d'histoire du Canada par l'abbé Albert Tessier à l'intérieur d'un certificat de pédagogie en 1941-1942. Ce même Maheux a entre-temps obtenu en 1938 la chaire d'histoire du Canada. Il prononcera à ce titre quelques conférences publiques données de façon très irrégulière à l'École normale supérieure jusqu'en 1947. Il poursuivait en cela une glorieuse tradition inaugurée au XIX ${ }^{\mathrm{e}}$ siècle par Jean-Baptiste Ferland (1858-1862) et Charles-Honoré Laverdière (1862-1872), et avec laquelle Chapais avait renoué quelques années auparavant ${ }^{11}$. À l'Université de Montréal, Lionel Groulx présente depuis 1915 une série de cinq ou six cours publics sur une période ou sur un thème de l'histoire du Canada. Ouvertes au grand public, publicisées trompettes sonnantes par Omer Héroux dans les pages du Devoir, ces conférences font affluer tout ce que Montréal compte de notables culturels. Les auditoires sont parfois fort nom-

9. Archives de l'Université de Montréal. Fonds du secrétariat général, D35 (1876-1950). Faculté des lettres /838. (Rapports, mémoires, 1929).

10. Ibid.

11. Sur la place de l'histoire à l'Université Laval voir: Annuaire de l'Université Laval (1920+); Marcel Trudel, Mémoires d' un autre siècle, op. cit., 313 p.; Marcel Trudel, «Les débuts de l'Institut d'histoire à l'Université Laval», Revue d'histoire de l'Amérique française, 27,3 (décembre 1973): 397-402; Maurice Lebel, «Thèses présentées à la Faculté des Lettres de l'Université Laval (1940-1947)», Culture, 8,1 (mars 1947): 67-75; Jean Hamelin, Histoire de l'Université Laval. Les péripéties d'une idée (Sainte-Foy, Les Presses de l'Université Laval, 1995), $341 \mathrm{p}$. 
breux: 1200 personnes lors de la conférence inaugurale patronnée par $\mathrm{M}^{\mathrm{gr}}$ Bruchési ${ }^{12}$. Nous sommes loin du séminaire de recherche.

La Faculté des lettres de l'Université de Montréal, fondée en 1920 , est la première à offrir quelques cours fermés d'histoire à des étudiants dûment inscrits et qui ressortent diplôme en poche. Le programme d'études de 1920 conduit à deux licences, l'une de culture générale, l'autre d'enseignement ${ }^{13}$. La licence d'enseignement comporte elle-même deux sections: lettres-grammaire et histoire-géographie. Ce programme est refondu en 1929-1930 et séparé entre études de culture générale, qui conduisent à la licence ès lettres (sections lettres-grammaire ou histoire-géographie), et études de culture spéciale, qui mènent aux grades de maitrise et de doctorat ès lettres. L'obtention de ce dernier diplôme exige la préparation pendant au moins deux ans d'une thèse d'une valeur critique certaine ${ }^{14}$.

12. Lionel Groulx, Mes mémoires, 1: 1878-1920 (Montréal, Fides, 1970), 257-258.

13. Annuaire, Université de Montréal, Faculté des lettres (1920+).

14. L'obtention du diplôme de doctorat est loin d'être une simple formalité. Antoine Bernard, professeur d'histoire de l'Acadie à la Faculté des lettres, soumet en 1928 à l'approbation du doyen de la Faculté, $\mathrm{M}^{\mathrm{gr}}$ Émile Chartier, un projet de thèse de doctorat sur la vie et l'œuvre de Nicolas Denys, premier seigneur des côtes du golfe Saint-Laurent (1632-1668). L'année suivante Chartier demande à Aegidius Fauteux, bibliothécaire à la bibliothèque municipale de Montréal, d'évaluer le travail. Son verdict parvient le 15 mai: «En tant qu'histoire de l'Acadie, la thèse du Rév. Frère Bernard peut avoir une certaine valeur littéraire. Le récit est alertement mené, d'un style assez vivant et d'une lecture véritablement agréable. Mais sa valeur historique est beaucoup moins apparente. En dépit des promesses de l'auteur, dans son introduction, je ne crois pas que cette thèse puisse être considérée comme une véritable contribution à l'histoire acadienne. Elle ne me paraît jeter aucune lumière nouvelle sur les événements qui se sont déroulés en Acadie depuis 1605 jusqu'à 1710. Bien qu'elle réfère ici et là à certaines pièces d'archives par ailleurs connues, elle n'est qu'un réarrangement des travaux déjà imprimés sur le même sujet.» Le lendemain, Chartier écrit à Bernard: «Vu les conclusions des deux rapporteurs, le conseil croit sage de vous proposer l'attitude qui suit. Sans doute votre dissertation ressemble à toutes celles que l'Université a acceptées jusqu'à présent; elle les égale, si elle ne leur est supérieure. Mais elle porte sur l'histoire du Canada, où les Anglais ont pris sur nous une telle avance et ont produit des études si personnelles et originales, qu'ils dauberaient peut-être sur une œuvre de simple vulgarisation. Vous avez d'ailleurs certains ennemis personnels qui attendent l'occasion de reprendre leur campagne: il ne faut pas leur donner prise. Or l'acceptation de la soutenance comporte implicitement l'autorisation d'imprimer et la consécration de l'œuvre tant par l'Université que par la Faculté. Le conseil estime donc que vous devriez prendre tout le temps nécessaire pour retoucher votre manuscrit d'après les indications de vos juges, le compléter par des découvertes originales, l'alléger d'une préface que je vous avais d'avance prié de supprimer, en un mot en faire une œuvre qui supporte les feux de la critique.» Bernard choisit alors un nouveau sujet - «L'influence prépondérante de l'Église catholique dans le relèvement du peuple acadien aux Provinces maritimes (1755-1933)» - , sa soutenance n'ayant lieu qu'en mai 1934 devant un jury bienveillant composé de Jean Bruchési, son collègue à la Faculté des lettres, et de Émile Dubois, curé de Saint-Janvier et ancien professeur d'histoire au séminaire de Sainte-Thérèse. L'épisode, qui met en jeu des considérations politiques, démontre néanmoins que les critères de l'histoire critique commencent à s'imposer dans le système universitaire. Archives de l'Université de Montréal, Fonds du département d'histoire, Dossiers particuliers, E 16/ D1, 2, 9. Antoine Bernard, c.s.v. (12 octobre 1921-2 mars 1949), Antoine Bernard à Émile Chartier, 28 mai 1928; Aegidius Fauteux à Émile Chartier, 15 mai 1929; Émile Chartier à Antoine Bernard, 16 mai 1929. 
Le programme d'études de la licence ès lettres, section histoiregéographie, comprend des cours de littérature (latine, grecque, française, anglaise et canadienne) et deux cours fermés d'histoire: Histoire du Canada, donné par Groulx, et le cours d'Histoire générale, fief laïque tenu tour à tour par de fortes personnalités qui feront carrière dans la diplomatie et la politique: Jean Désy (1920-1927), Jean Bruchési (1927-1937) et Jean-Marie Nadeau (1937-1948) ${ }^{15}$. Il faut ajouter les conférences publiques sur l'histoire canadienne-anglaise, données de 1927 à 1941 par William Atherton, titulaire de la chaire de littérature anglaise, et les cinq cours publics annuels d'histoire de l'Acadie assumés d'abord par le docteur Edmond Aucoin en 1923, puis par Antoine Bernard de 1926 à 1947 et dont le coût est payé par le financier et philanthrope Auguste Richard ${ }^{16}$. À ce groupe se joignit Guy Frégault en 1942-1943, comme chargé de cours en histoire et en littérature canadiennes.

L'idée de mettre sur pied un programme d'études historiques a été lancée à quelques reprises à Montréal et à Québec. Des obstacles pour ainsi dire structurels diffèrent le projet à plus tard. C'est que l'enseignement promu dans les facultés des lettres et des arts au Canada français donne la priorité, non pas à la spécialisation dans un champ donné, mais à l'acquisition d'une vaste culture générale. À la Faculté des lettres de l'Université de Montréal, le régime d'études prévoit que 580 leçons sont requises pour l'obtention de la licence ès lettres, sec-

15. Jean Désy (1893-1960): licencié en droit (Université Laval de Montréal) et en sciences économiques (École libre des sciences politiques, Paris, 1920). Professeur agrégé (1920), puis titulaire (1922) à la Faculté des lettres de l'Université de Montréal. Engagé au ministère des Affaires étrangères du Canada en 1925; postes diplomatiques à la SDN (1925), à l'ambassade de France (1928-1933), en Belgique et aux Pays-Bas (1933-1939); directeur du service international de Radio-Canada (1942-1944); ambassadeur au Brésil (1944), en Italie (1947) et en France (1953-1958). Jean Bruchési (1901-1979): licencié en droit (UDM, 1924); études en sciences politiques à Paris (École libre des sciences politiques, section diplomatique, 1925-1927). Professeur à la Faculté des lettres de l'Université de Montréal (1927-1937). Donne parallèlement le cours de politique économique à la Faculté des sciences sociales. Sous-secrétaire de la province de Québec (1937-1959), puis ambassadeur en Espagne (1959). Jean-Marie Nadeau (1906-1960): licencié en droit (UDM, 1930) et en lettres-histoire (Université de Rennes); remplace Bruchési, à la fois à la Faculté des lettres (1938-1948) et à la Faculté des sciences sociales; s'engage dans la lutte politique en 1944 au sein du parti libéral du Québec; candidat défait dans le comté de Rouville (1948); candidat défait à la chefferie du parti en 1951 contre Georges-Émile Lapalme; chef de la commission politique du parti libéral de 1952 jusqu'à sa mort en 1960.

16. Le chapitre montréalais de la Société L'Assomption, société nationale acadienne, obtient en 1929 de la Faculté des lettres que les conférences publiques sur l'histoire de l'Acadie soient tenues le dimanche après-midi, et non les mercredis et les vendredis en soirée, dans les locaux même de l'association afin d'attirer le plus d'auditoire possible. Archives de l'Université de Montréal, Fonds du département d'histoire, Dossiers particuliers, E 16/ D1, 2, 9. Antoine Bernard, c.s.v. (12 octobre 1921-2 mars 1949). Hervé Richard à Émile Chartier, 27 novembre 1929; J. L. Buster (?) à Émile Chartier, 10 décembre 1929. 
tion histoire-géographie, dont 180 en histoire. Chaque leçon dure 55 minutes. C'est assez peu pour prétendre à la spécialisation. La francophilie de l'époque, inscrite dans les structures institutionnelles, renforce cette tendance généraliste: les programmes d'études et les grades universitaires des facultés de lettres sont harmonisés avec le modèle français de la licence ès lettres (fondé sur le principe du cumul des certificats ${ }^{17}$ ). Même les bourses d'études du secrétariat de la province, les seules auxquelles ont accès les étudiants qui veulent poursuivre des études avancées, limitent l'inscription aux institutions européennes, principalement françaises. Ce n'est qu'en 1937 que la règle sera modifiée afin de permettre la fréquentation des universités américaines où les études spécialisées sont mieux établies ${ }^{18}$.

De plus, la clientèle visée par les facultés des lettres - les maîtres qui se dirigent vers l'enseignement secondaire ou les professeurs des collèges classiques - indique qu'on est assez peu préoccupé de former des chercheurs ou des spécialistes. Comme l'enseignement dans les collèges classiques est réservé aux prêtres séculiers et aux religieux, la clientèle laïque se fait plutôt rare: des fils de bonne famille qui entreprennent des études désintéressées, des professionnels et des fonctionnaires qui viennent y renouveler leur capital culturel, des femmes qui se cherchent mari, comme l'on disait méchamment à l'époque $^{19}$. Et aussi quelques passionnés, aux velléités littéraires, tels Frégault et Trudel qui, par une reconversion vers l'histoire, aspirent à une carrière universitaire. Marcel Fournier a émis l'hypothèse selon laquelle cette persistance dans la promotion des idéaux humanistes et dans la diffusion d'une culture savante aurait correspondu à une stratégie de la classe supérieure canadienne-française, idéologiquement et politiquement dominante, visant à perpétuer un ordre culturel et un programme d'enseignement qui avait assuré jusque-là son maintien au sommet $^{20}$. L'idée est séduisante. Quoi qu'il en soit, les diplômés sont rares: 55 étudiants obtiennent la licence ès lettres à l'Université Laval

17. La licence ès lettres de la Faculté des lettres de l'Université Laval est calquée, lors de sa création en 1937, sur le modèle de licence en Sorbonne (4 certificats d'études supérieures: latin, grec, français et un quatrième au choix). Voir Marcel Trudel, op. cit., 127. À Montréal, la licence, qui exige de suivre au moins un cours dans toutes les matières, s'en inspire fortement. Par ailleurs, une entente d'équivalence du grade de licence ès lettres de l'Université de Montréal avec le diplôme de licence d'une université française, a été arrêtée avec le ministère de l'Instruction publique et des Beaux-arts de France en juillet 1922. Voir Annuaire 1951-1952, Université de Montréal, Facultés des lettres, 19.

18. Yves Gingras, Pour l'avancement des sciences. Histoire de l'ACFAS 1923-1993, (Montréal, Boréal, 1994), 70.

19. Marcel Trudel, op. cit., 127. Rappelons tout de même que c'est ainsi que Liliane Rinfret a rencontré Guy Frégault, son futur mari.

20. Marcel Fournier, «Le frère Marie-Victorin et les petites sciences», loc. cit., 76-78. 
(dont 15 laïques) de 1920 à $1939^{21}$. À l'Université de Montréal, seulement 25 étudiants sont inscrits à la Faculté des lettres en $1943^{22}$. Difficile, avec de si faibles effectifs étudiants, d'organiser un enseignement spécialisé en histoire. À cela s'ajoutent des problèmes financiers, liés à la crise des années 1930, qui freinent l'expansion des universités, amènent la suspension des travaux de construction de l'Université de Montréal et mettent même sa survie en question ${ }^{23}$.

Il est cependant un lieu où s'amorce un enseignement spécialisé en histoire et où s'organisent des activités de recherche: l'Institut d'études médiévales Albert-le-Grand. Rattaché à la Faculté de philosophie de l'Université de Montréal en 1942, l'Institut, fondé en 1931 à la suggestion d'Étienne Gilson, fut d'abord érigé comme une composante du couvent d'études d'Ottawa, collège philosophique et théologique des dominicains canadiens ${ }^{24}$. L'un des objectifs invoqués lors de la fondation est la nécessité de «former des étudiants aux disciplines et aux méthodes de l'histoire ${ }^{25}$ ». Mais «le but de l'Institut est doctrinal», spécifie-t-on dans l'annuaire général de l'Université de Montréal en 1946-1947, l'histoire n'étant associée à la doctrine que

21. Marcel Trudel, op. cit., 127.

22. Hélène-Andrée Bizier, L'Université de Montréal. La quête du savoir (Montréal, Libre Expression, 1993), 175.

23. À deux reprises, soit en février 1932 et en juin 1933, l'Université de Montréal suspend le versement des salaires du personnel, y compris ceux des professeurs. Le salaire annuel de Lionel Groulx comme professeur est amputé d'un versement mensuel de $240 \$$, le nombre de versements passant de 10 à 9 (pour un total de $2160 \$$ plutôt que de $2400 \$$ ) et ce, durant 13 ans (1932-1945). Pourtant, le contrat d'embauche de 1928 stipulait bien la somme annuelle de 2400 \$, contre laquelle Groulx s'engageait à «consacrer tout son temps à l'enseignement et à la recherche, à renoncer à la direction de l'Action française, à donner chaque année son cours fermé de 30 leçons, à prononcer chaque année 6 conférences publiques exposant la substance de ses recherches et à donner 4 leçons annuelles sur la méthodologie de l'histoire du Canada» (cours de perfectionnement à l'intention du personnel des collèges classiques affiliés à la Faculté des arts de l'Université de Montréal, pratique qui fut abandonnée en 1940 lorsque s'ouvrit l'École normale secondaire). Archives de l'Université de Montréal, Fonds du secrétariat général, D35 (1876-1950), Faculté des lettres /833. Personnel enseignant, 1920-1950. «Projet de résolution et projet de contrat avec l'abbé Groulx. Extrait du procès-verbal de la $56^{\mathrm{e}}$ réunion du Conseil de la Faculté des lettres de l'Université de Montréal, tenue le vendredi 24 février 1928, au bureau du doyen»; «Extrait du procès-verbal de la $395^{\circ}$ réunion régulièrement convoquée, de la Société d'administration de l'Université de Montréal, tenue le 19 décembre 1949, à 3:30 de l'après-midi, au bureau de la Société. 4193. Budget 1949-1950. Faculté des lettres - Supplément voté re: pension du chanoine Groulx».

24. Gilson avait participé l'année précédente à la fondation d'un institut semblable à Toronto (rebaptisé du nom d'Institut pontifical d'études médiévales au début des années 1940). Voir Louis-Marie Régis, «L'Institut d'études médiévales de Montréal», La Revue dominicaine, 48,2 (juillet-aôut 1942): 112. 170.

25. «L'Institut d'études médiévales d'Ottawa», La Revue dominicaine, 37 (mars 1931): 
comme «première auxiliaire ${ }^{26}$ ». L'examen du contexte de la fondation de l'Institut démontre que les enjeux de la lutte politique, idéologique et doctrinale prennent le pas sur les visées «scientifiques».

Il se trouve que la mise sur pied de cet Institut s'inscrit dans une stratégie de pouvoir et d'influence de l'ordre des dominicains canadiens à l'intérieur même de la structure de l'Église ${ }^{27}$. Rappelons que les dominicains proposent une relecture de saint Thomas d'Aquin et une volonté de présence au monde moderne dans le respect de la tradition. Absents jusqu'alors du système d'éducation, ils disposent de peu de moyens pour transmettre leurs idées. Ils ne souhaitent pas œuvrer dans les collèges classiques où se trouvent déjà d'autres communautés, mais cherchent plutôt à conquérir, dans l'enseignement supérieur, une place forte dans les différents domaines du haut savoir: philosophie avec l'entrée du père Ceslas-Marie Forest à la Faculté de philosophie de l'Université de Montréal, sciences sociales avec l'envoi du père Georges-Henri Lévesque pour un séjour d'études en Europe, doctrine et histoire médiévales avec la fondation de l'Institut d'études médiévales. Le rattachement éventuel de l'Institut à l'Université d'Ottawa, dirigée par les oblats, pose problème. Outre l'obstacle de la coexistence de deux ordres concurrents, la réputation de «libéraux» des dominicains effraie. C'est que l'Institut gravite autour de l'école dominicaine du Saulchoir où s'est amorcée, notent Jean Hamelin et Nicole Gagnon, «une révolution épistémologique cruciale: la substitution de la méthode historique à la méthode déductive dans la réflexion théologique ${ }^{28}$ ». Or, les oblats qui dirigent l'Université d'Ottawa ne sont pas prêts à accueillir une innovation du genre. C'est donc au père Forest, qui occupe depuis 1926 le poste stratégique de doyen de la Faculté de philosophie de l'Université de Montréal, qu'échouera la tâche de doter l'Institut du statut universitaire qui lui manquait.

Par-delà la position des dominicains au sein de la structure ecclésiale, c'est la place même de l'Église au sein de la société qui est en jeu. Le développement des philosophies modernes, «nominalistes, évolutionnistes et pragmatiques [...] a sapé les fondements de la morale et du droit», argue le doyen Forest ${ }^{29}$. Il importe donc de leur

26. Louis-Marie Régis, «L'Institut d'études médiévales», Annuaire général 1948-1949. Université de Montréal (28 année): 346.

27. Voir Jean Hamelin et Nicole Gagnon, «Le $\mathrm{XX}^{\mathrm{e}}$ siècle», dans Nive Voisine, dir., Histoire du catholicisme québécois, 1: 1898-1940 (Montréal, Boréal Express, 1984): 414-419; Catherine Pomeyrols, Les intellectuels québécois: formation et engagements, 1919-1939 (Montréal, L'Harmattan, 1996), 140.

28. Nive Voisine, dir., op. cit., 416.

29. «Réorganisation de la Faculté de philosophie de l'Université de Montréal», La Revue dominicaine, 48,2 (juillet-août 1942): 105. 
opposer une doctrine philosophique ferme, la «philosophie chrétienne traditionnelle». Or, cette doctrine, où atteint-elle la plénitude de son développement? Au Moyen Âge, poursuit le directeur de l'Institut, Louis-Marie Régis, alors que s'élabore la synthèse thomiste, cet aboutissement de la pensée chrétienne ${ }^{30}$. L'Institut ne retourne pas à cette période de l'histoire «pour le simple plaisir de revenir au passé, enchaîne Régis, mais bien à cause de sa valeur idéologique et christianisante unique ${ }^{31} »$. En outre, l'étude historique de l'antiquité chrétienne ne doit pas être "abandonnée aux adversaires du catholicisme», et elle revêt en cela une importance stratégique pour l'Église. En France, après la tourmente révolutionnaire qui supprima de nombreux ordres religieux, le catholicisme français ne sut pas lutter efficacement contre le rationalisme et le positivisme. «Il ne faudrait pas recommencer sur le terrain du Moyen Âge cette lamentable expérience, et venir au travail après que d'autres l'auraient engagé et orienté contre notre foi ${ }^{32}$.»

L'application rigoureuse des méthodes de la critique historique pour l'étude des doctrines philosophiques, théologiques et canoniques n'apparaît nullement incompatible avec cet objectif. L'article 2 de la Constitution apostolique Deus Scientiarum Dominus de mai 1931, qui impose de nouveaux règlements aux facultés canoniques du monde entier, demande que les étudiants catholiques soient «formés à la connaissance des sources, et à la pratique des recherches et des travaux scientifiques ${ }^{33}{ }^{\prime}$. Les dirigeants de l'Université de Montréal et de la Faculté de philosophie, qui feront approuver par Rome en 1936 la nouvelle organisation des études, ne prétendent donc que se conformer aux directives du pape en rattachant un institut de recherche et d'enseignement spécialisé en histoire des doctrines médiévales ${ }^{34}$.

Ces réserves faites, l'histoire n'en occupe pas moins une place importante dans les programmes d'études. Les cours de l'Institut cou-

30. «Tout ce que l'antiquité avait produit de meilleur, c'est-à-dire l'humanisme grécoromain, les illustres penseurs du moyen âge l'ont, en effet, couronnée par la création de la "disciplina sacra", synthèse géniale de tout l'apport philosophique ancien et de la doctrine évangélique, apportant ainsi à l'humanité, la seule vision intégrale de l'univers terrestre»: L.-M. Régis, «L'Institut d'études médiévales», La Revue dominicaine, 48,2 (juillet-août 1942): 109110 .

31. Louis-Marie Régis, «L'Institut d'études médiévales», Annuaire général 1948-1949. Université de Montréal (28 $8^{\mathrm{e}}$ année): 345.

32. «L'Institut d'études médiévales», La Revue dominicaine, 37 (1931): 167.

33. «L'Institut d'études médiévales», Annuaire général 1947-1948. Université de Montréal (27 année): 89.

34. Voir Ceslas-Marie Forest, «Vingt-cinq ans de philosophie à l'Université de Montréal», Extrait des activités philosophiques 1945-1946, 13; Louis-Marie Régis, «Institut d'études médiévales», Annuaire général 1947-1948. Université de Montréal (27 année): 88-89. 
vrent un cycle de trois années et conduisent aux diplômes de baccalauréat, de licence et de doctorat ès sciences médiévales. La première année consiste en un enseignement général sur l'histoire des idées et des institutions du Moyen Âge. Dans cette perspective, des cours d'initiation à l'histoire générale du Moyen Âge et des séances d'explication de textes et des exercices pratiques sont inscrits au programme, afin que l'étudiant s'imprègne des doctrines philosophiques, théologiques et canoniques ${ }^{35}$. Les deuxième et troisième années sont consacrées à un enseignement technique et pratique orienté vers l'étude des textes, l'étudiant devant se spécialiser soit en philosophie, soit en théologie.

Le sérieux du programme d'enseignement est attesté par des règles d'admission très exigeantes: les étudiants doivent être détenteurs d'un baccalauréat ès arts et connaître le français, l'anglais, le latin et l'allemand (qu'ils s'engagent à apprendre s'ils ne le connaissent pas déjà). Ils doivent également absorber, durant leurs études, une forte dose d'érudition, dans des disciplines variées et se familiariser avec des techniques pointues: paléographie, archéologie, historiographie, linguistique, etc. Les inscrits sont peu nombreux - une vingtaine en 1949, dont une moitié d'étrangers - et les séminaires sont limités à sept ou huit étudiants ${ }^{36}$.

Le groupe de professeurs et de chargés de cours est composé d'érudits formés dans les universités européennes et américaines: la Sorbonne, l'École des Chartres, l'Institut biblique de Jérusalem, les universités d'Oxford, de Heildelberg, de Rome et de Fribourg. Parmi les chargés de cours étrangers de renom, mentionnons le père MarieDominique Chenu, régent des études au Saulchoir, qui enseignera à l'Institut de 1931 à 1935 et Henri-Irénée Marrou, de passage à l'automne de $1949^{37}$. L'œuvre de l'Institut est complétée par une bibliothèque de plus de 6000 volumes et par un service de publications scientifiques. La convoitise dont fait l'objet l'Institut d'Ottawa de la part des évêques de Montréal, $\mathrm{M}^{\mathrm{gr}}$ Georges Gauthier et $\mathrm{M}^{\mathrm{gr}}$ Joseph Charbonneau, n'est sans doute pas étrangère à l'œuvre d'édition de

35. Annuaire général 1947-1948. Université de Montréal (27e année): 88 et ss; Benoît Lacroix, «L'Institut d'études médiévales», L'Action universitaire, 14,2 (juillet 1948): 364-367.

36. Benoît Lacroix, loc. cit., 366; Benoît Lacroix, «Pourquoi le moyen âge», La Revue dominicaine, 55,1 (janvier 1949): 152-153.

37. Archives de l'Université de Montréal. Fonds du Secrétariat général D 35 (18761950). Faculté de Philosophie /810, «Programmes de cours, 1931-1950 de l'Institut d'études médiévales». Voir également Benoît Lacroix, «L'institut d'études médiévales», L'Action universitaire, 14,2 (juillet 1948): 365. 
l'Institut, notamment la publication en 1941 de la Somme de théologie de saint Thomas d'Aquin, qui fera sa renommée ${ }^{38}$.

Enfin, les propagandistes de l'Institut reconnaissent la fin doctrinale de cette institution, tout en spécifiant que c'est par la critique historique qu'ils entendent y parvenir. Certes, il s'agit de célébrer le Moyen Âge et le thomisme comme sommets de la pensée chrétienne, mais ils s'engagent parallèlement à «faire connaître le vrai Moyen Âge», qui n'a que très peu de parenté avec les Dark Ages qu'on a imaginés, et le vrai thomisme, non cette doctrine rapiécée des manuels de synthèse ${ }^{39}$. Or, pour saisir les doctrines médiévales dans leur véritable sens, explique Louis-Marie Régis, il faut connaître «leur langage et leur vocabulaire technique», il faut connaître «leur contexte noétique», «les doctrines de fond qui commandent toute la structure de leur pensée», il faut approfondir les conditions historiques de leur production, il faut, en somme, se faire historien ${ }^{40}$.

Néanmoins, le bilan du développement de l'histoire dans l'enseignement supérieur avant 1945 au Canada français n'impressionne pas. Certes, l'histoire fait son entrée à l'université, mais elle y est éparpillée dans différentes facultés, sans programme propre, dominée par les exigences de la reconduction du programme d'enseignement des humanités classiques, associée aux lettres et à la philosophie comme auxiliaire et subordonnée aux enjeux de la lutte doctrinale et idéologique. Cette indigence du développement des études historiques est comblée en partie par la multiplication, au même moment, des sociétés savantes ${ }^{41}$, celles-ci devenant le lieu d'élaboration privilégiée de l'historiographie canadienne-française de l'entre-deux-guerres.

\section{L'HISTOIRE AU SEIN DES SOCIÉTÉS SAVANTES}

Les années 1920 marquent le départ d'un mouvement de création de sociétés savantes consacrées, en tout ou en partie, à l'étude du passé: à la Société royale du Canada, qui regroupe déjà bon nombre

38. Doctoris communis Sancti Thomae De Aquino Summa Theologica, cura et studio Instituli studiorum medievalium ottaviensis, ad textum sancti Pii PP V jussu confectum recognita. Voir «Une édition de saint Thomas au Canada», Culture, 2,2 (mars 1941): 129-153.

39. Benoît Lacroix, «Pourquoi le moyen âge», La Revue dominicaine, 55,1 (janvier 1949): 152-161.

40. «L'Institut d'études médiévales», L'Action universitaire, 8 (septembre 1942): 27-28.

41. La définition de la société savante la plus complète, retenue par Jean-Pierre Chaline, est celle de La grande encyclopédie du XIX siècle: "La société savante [...] est une réunion d'hommes de sciences, de lettrés, d'érudits, de penseurs, qui mettent en commun leurs efforts, leur savoir et leurs ressources en vue de faire progresser ou prospérer la branche des connaissances humaines à laquelle ils se sont spécialement adonnés ou qui les intéresse plus particulièrement», Jean-Pierre Chaline, Sociabilité et érudition. Les sociétés savantes en France (Paris, Éditions du Comité des travaux historiques et scientifiques, 1995), 20. 
d'historiens, s'ajoutent à partir de 1926 une vingtaine de sociétés d'histoire locale, ainsi que quelques associations plus spécialisées, telles que la Société canadienne d'histoire de l'Église catholique et la Société des dix. Le rôle de ces groupements n'est pas négligeable dans la genèse d'un espace de production et de reconnaissance propre à l'histoire: ils participent à sa délimitation en fixant les frontières entre l'histoire et la littérature ou l'engagement ${ }^{42}$; ils rompent l'isolement quasi total dans lequel les historiens étaient auparavant plongés; ils favorisent la mise en place d'un système d'interrelations entre individus réunis par une pratique commune - l'écriture de l'histoire fondé sur l'échange et la reconnaissance entre pairs; ils permettent l'acquisition d'habitus disciplinaires en l'absence de programme de formation universitaire ${ }^{43}$. L'exemplification de ces fonctions passe par un examen attentif des différents groupements d'historiens.

\section{La Société royale du Canada}

La Société royale du Canada constitue, depuis sa fondation en 1882, le regroupement par excellence des intellectuels canadiensfrançais et ce, jusqu'à la Deuxième Guerre mondiale ${ }^{44}$. Inspirée de la Royal Society de Londres et de l'Institut de France, la création de la Société royale vise à encourager le développement des lettres et des sciences au Canada. Quatre sections distinctes sont alors créées: I -Littérature française, histoire, archéologie; II - Littérature anglaise, histoire, archéologie; III - Mathématiques, sciences physiques et chimiques; IV - Géologie et biologie. Une cinquième section, consacrée à la biologie, s'ajoute en 1918. Pour favoriser la diffusion des travaux de la Société, le gouvernement fédéral participe au financement d'un

42. Sur le rôle des associations littéraires dans la constitution du champ littéraire au Québec, voir Pierre Rajotte, «Les associations littéraires au Québec (1870-1895). De la dépendance à l'autonomie», RHAF, 50,3 (hiver 1997): 375-400.

43. Marcel Trudel, Pierre Savard et Fernand Ouellet ont souligné, ailleurs, l'importance de ces premiers groupements: Marcel Trudel, «Les dix ans de l'Institut», RHAF, 10,1 (juin 1956): 3-12; Pierre Savard, «L'histoire de 1900 à 1930» et «L'histoire de 1930 à 1945», dans Pierre de Grandpré, Histoire de la littérature française du Québec (Montréal, Beauchemin, 1969), II: 134-148; IV: 306-316; Fernand Ouellet, «L'émergence dans le Canada du XX' ${ }^{\mathrm{e}}$ siècle de l'histoire comme science sociale», Mémoires de la Société royale du Canada, 4,20 (1982): 35-81.

44. Pour une histoire générale de la Société royale du Canada, voir Carl Berger, Honour and the Search for Influence. A History of the Royal Society of Canada (Toronto, University of Toronto Press, 1996), 167 p. Voir également Yves Lamarche, «Le champ intellectuel et la structure de ses positions: l'exemple de la Société royale du Canada», Sociologie et Sociétés, 7,1 (mai 1975); Victor Morin, «Les origines de la Société royale», Les Cahiers des dix, 2 (1937): 157-198; pour la section des sciences physiques et chimiques: Yves Gingras, Les origines de la recherche scientifique au Canada. Le cas des physiciens (Montréal, Boréal, 1991), chapitre IV; pour la section française: Pierre Rajotte, loc. cit. 
périodique annuel qui se veut le véhicule de la science et des lettres canadiennes: Les mémoires et comptes rendus de la Société royale du Canada.

La participation des historiens à la Section I est importante: plus du tiers des membres entre 1920 et 1945 se sont illustrés par la production d'ouvrages historiques ${ }^{45}$, le sommet ayant été atteint en 1940 avec 16 historiens sur 36 membres $(44,4 \%)$. Cette part déclinera par la suite, pour n'atteindre que $28,3 \%$ en 1950 (13 historiens sur 46). Cet espace n'est donc pas proprement historien: la majorité des membres sont des littéraires. L'utilisation du terme même d'historien pose problème. Parmi les membres qui peuvent s'enorgueillir d'une production historiographique, on trouve des spécialistes d'autres disciplines (le sociologue Léon Gérin, l'anthropologue Marius Barbeau), des archivistes (Francis J. Audet, Séraphin Marion, Ivanhoë Caron, PierreGeorges Roy, Édouard-Zotique Massicotte, Gustave Lanctot, Antoine Roy), des juristes (le notaire Victor Morin, le juge L.-A. Prud'homme), des hommes politiques (Jean-Marie Nadeau, les sénateurs Rodolphe Lemieux et Thomas Chapais), des écrivains de profession (Léo-Paul Desrosiers, Jean Bruchési), des abbés aux activités multiples (Albert Tessier, Joseph-Élie Auclair, Georges Robitaille), des professeurs d'université (Lionel Groulx, Arthur Maheux et Georges Simard). La production écrite de la plupart de ces membres n'est pas non plus exclusivement historique: certains ont écrit des romans (Groulx, Desrosiers), de la poésie (Massicotte), nombre d'entre eux ont pratiqué le journalisme et dirigé des périodiques ${ }^{46}$. L'admission à

45. Selon Pierre Savard, une règle non écrite veut que l'on soit l'auteur d'au moins un livre pour être élu à la Société. Ainsi, Arthur Maheux, qui a posé sa candidature en 1943, s'apercevant qu'il ne rencontrait pas les critères d'admission, aurait ramassé quelques conférences de ses fonds de tiroirs et fait faire presto un livre aux presses de l'Action sociale de Québec. Pierre Savard, «Gustave Lanctot et la Société royale du Canada», Les Cahiers des dix, 48 (1993): 238-239.

46. Parmi ces «historiens» qui ont pratiqué le journalisme et ont dirigé des revues, mentionnons (avec entre parenthèses l'année d'admission à la SRC): l'abbé Joseph-Élie Auclair (1919), rédacteur à la Semaine religieuse (1905-1922) et directeur de la Revue canadienne (1908-1922); Thomas Chapais (1902), rédacteur en chef du Courrier du Canada (1884-1901), rédacteur de chroniques historiques dans La Presse de 1897 à 1911 sous le pseudonyme d'Ignotus, rédacteur d'une chronique à la Revue canadienne (1899-1922), intitulée des «Faits et des œuvres»; Léo-Paul Desrosiers (1942), chroniqueur parlementaire à Ottawa pour le journal $L e$ Devoir (1920-1928) et auteur de nombreux romans historiques; Aegidius Fauteux (1918), chroniqueur parlementaire à Ottawa pour La Patrie de 1903 à 1908, journaliste à La Presse (19081918) et rédacteur en chef (1909-1912); Lionel Groulx (1918), directeur à L'Action française (1920-1928); Gustave Lanctot (1926), journaliste au Canada et à La Patrie (1907-1909); l'abbé Arthur Maheux (1941), directeur du Canada français (1919-1921; 1938-1942); Édouard-Zotique Massicotte (1920), collaborateur entre 1895 et 1900 à l'Écho des jeunes, au Recueil littéraire, tient une chronique à L'Étendard et au Monde illustré dont il devient directeur en 1898; Jean-Marie Nadeau (1946), chroniqueur à L'Ordre d'Olivar Asselin (1934-1935) et directeur du 
la Section I ne consacre donc pas tel membre comme historien et tel autre comme poète, mais reconnaît plutôt sa contribution aux lettres canadiennes-françaises, l'histoire constituant un sous-genre de l'activité littéraire considérée dans son acception la plus large. Cette indistinction entre littérature de fiction et production savante ne sera d'ailleurs pas sans provoquer quelques appels pour une meilleure représentation des sciences sociales au sein de la Société royale ${ }^{47}$. Néanmoins, la Section I constitue avant 1930 le seul lieu réunissant un groupe d'historiens où ceux-ci peuvent échanger et discuter.

Les réunions annuelles de la Société royale et de la Section I ont lieu dans la troisième semaine de mai et durent trois jours. Des travaux sont alors lus par des membres comme par des non-membres. Un comité de lecture et de publication a pour mission de juger les communications méritant d'être reproduites dans les Mémoires de la Sociétét $^{48}$. Le nombre important d'études à caractère historique qui y sont publiées entre 1920 et 1950 - plus de 150 - témoigne de l'engouement des sociétaires pour le passé et, de façon plus générale, de la place de l'histoire dans les lettres canadiennes. Le sommet est atteint durant les années 1920 et 1930, alors que plus de $60 \%$ de tous les mémoires publiés peuvent être qualifiés d'historiques, la proportion chutant à $35 \%$ durant les années $1940^{49}$. Les sujets sont souvent à

Canada (1951); Pierre-Georges Roy (1911), collaborateur dans les années 1890 au Quotidien de Lévis, au Canadien et à L'Action catholique à Québec, fondateur en 1890 d'une revue littéraire à laquelle É.-Z. Massicotte participe, Le Glaneur, puis éditeur-directeur du Bulletin des recherches historiques de 1895 à 1942, fonctions reprises par son fils, Antoine Roy (1948).

47. Des pressions seront faites en ce sens dès 1908 par deux membres de la Section $I$, le sociologue et historien Léon Gérin et l'économiste Errol Bouchette. Voir Carl Berger, op. cit., 69-71.

48. Seulement une fraction des travaux lus à la séance annuelle sont publiés: 4 sur 12 en 1920, 4 sur 13 en 1921, 10 sur 18 en 1922, etc. Voir les Mémoires et comptes rendus de la Société royale du Canada, $3^{\mathrm{e}}$ série $(1920+)$.

49. Distinguer, à des fins de classement, les travaux qui relèvent du domaine propre de l'histoire est une entreprise malaisée. En effet, selon nos catégories actuelles, plusieurs des études qui y sont présentées se situent aux frontières des disciplines traditionnelles. L'étude du R. P. Hugolin sur Un peintre de renom à Québec en 1670: le diacre Luc François, récollet (1932), appartient-elle au champ de l'histoire de l'art, où relève-t-elle du domaine de l'histoire générale? Où la classer? Lorsque Marius Barbeau se penche sur Deux cent ans d'orfèvrerie chez nous (1939), fait-il œuvre d'historien ou d'ethnologue? De plus, il est courant de penser une production intellectuelle dans la spécificité de l'histoire de son genre ou de sa discipline et d'en explorer la dimension diachronique. Les dix études de $\mathrm{M}^{\mathrm{gr}}$ Émile Chartier sur l'histoire des lettres canadiennes et sur La vie de l'esprit au Canada français (1931-1939), appartiennent-elles au champ des études littéraires ou à celui de l'histoire intellectuelle? Il nous est donc apparu impossible d'établir des catégories mutuellement exclusives. Nous avons préféré colligé tous les travaux à caractère historique. Par cette méthode, nous sommes parvenu à des chiffres impressionnants: 54 mémoires à caractère historique sur $88(61,4 \%)$ durant les années 1920, 72 sur 117 $(61,5 \%)$ durant les années 1930,35 sur $99(35,4 \%)$ durant les années 1940 . Durant cette dernière décennie, un nombre beaucoup plus important de travaux proprement littéraires sont publiés (poèmes, critiques littéraires, études littéraires, etc.), alors que les historiens semblent désormais privilégier d'autres lieux d'élaboration et de publication. 
caractère anecdotique: biographie d'intendants, de gouverneurs, de membres du clergé et d'hommes politiques, monographies régionales, paroissiales ou familiales, rappel de l'action héroïque des premiers colons ou missionnaires, etc. ${ }^{50}$. Pour s'assurer que la Société se développe comme un lieu de production littéraire et scientifique, les règlements prévoient l'exclusion des membres qui n'auraient pas, pendant trois années consécutives, participé aux travaux de la société. Le règlement semble toutefois avoir été appliqué avec souplesse, les procès-verbaux de la Section I étant remplis de résolutions accordant un délai à tel ou tel membre pour s'y conformer ${ }^{51}$. On propose même en 1923 de porter ce délai à 5 ans et que soit conservée l'alternative entre l'obligation du travail et l'obligation de la présence ${ }^{52}$. La participation est variable, en fonction du lieu où se tient la réunion annuelle, les rencontres tenues à Montréal, Québec ou Ottawa étant les plus courues. Mais de façon générale, cette participation ne cesse de diminuer tout au long de la période étudiée: la présence passe d'une moyenne de $48 \%$ durant les années 1920 (18 membres), à $41 \%$ durant les années 1930 (16 membres) et à 35\% durant les années 1940 (14 membres), alors que la Société, soucieuse d'attirer l'attention du public et d'accroître son prestige, parcourt le pays d'un océan à l'autre (réunions à Halifax, Québec, Montréal, Kingston (2), Toronto (2), Lon-

50. Les mémoires publiés en 1932 sont typiques de cette production. Mentionnons: «La nomination des évêques catholiques de Québec sous le Régime anglais» par l'abbé Ivanhoë Caron; «Notes et documents concernant les gouverneurs d'Ailleboust, de Lauzon et de LauzonCharny» par $\mathrm{M}^{\mathrm{gr}}$ Amédée Gosselin; «William Brown (1737-1789). Premier imprimeur, journaliste et libraire de Québec. Sa vie et ses œuvres» par Francis-Joseph Audet; «Recherches historiques sur les spectacles à Montréal de 1760 à 1800» par É.-Z. Massicotte; «SaintVincent-de-Paul de l'île Jésus. Étude historique présentée à la Société royale du Canada» par l'abbé Élie-J. Auclair. Mémoires et comptes rendus de la Société royale du Canada, $3^{\mathrm{e}}$ Série, 26 (1932): iii.

51. Par exemple, Lionel Groulx qui a participé régulièrement aux travaux de la Société durant les années qui ont suivi son élection (présences en 1918, 1920, 1923, 1926, 1928, 1930), n'y reparaîtra plus de 1930 à 1952, année de sa démission, même lorsque la réunion annuelle est tenue à Montréal (comme en 1939 et en 1944): «Rapports de la Section I», Mémoires et comptes rendus de la Société royale du Canada, $3^{e}$ série (1915+). L'application stricte des règlements à la fin du XIX $\mathrm{XIèc}^{e}$ se s'est toutefois traduite, dans plusieurs cas, par la présentation de contributions de qualité douteuse. Daniel Mativat rapporte que plusieurs sociétaires ont produit des communications ne dépassant pas dix lignes. Daniel Mativat, Le métier d'écrivain au Québec (1840-1900) (Montréal, Triptyque, 1996), 419. Cité dans Pierre Rajotte, loc. cit., 390. Berger rappelle également la présentation d'un mémoire par É.-Z. Massicotte consistant en la présentation du recensement de Montréal de 1741 et l'énumération des 1474 noms, maison par maison, rue par rue. Voir Mémoires et comptes rendus de la Société royale du Canada, $3^{\mathrm{e}}$ série, 15 (1921); Carl Berger, op. cit., 34.

52. «Procès-verbal de la Section I», Mémoires et comptes rendus de la Société royale du Canada, 3e Série, 17 (1923): xxix. 
don, Vancouver). En fait, les membres les plus actifs et les plus assidus sont des historiens qui vivent et travaillent à Ottawa où sont basés les bureaux de la Société: Gustave Lanctot, Séraphin Marion et F.-J. Audet, archivistes aux Archives nationales à Ottawa, Léon Gérin, chef du bureau de traduction à la Chambre des communes (1917-1935) et, dans une moindre mesure, les sénateurs Rodolphe Lemieux et Thomas Chapais $^{53}$. Ces animateurs de la Section I se situent pour la plupart en marge du nationalisme, lorsqu'ils ne s'en font pas les critiques féroces. Laïques et bilingues, bien informés des réalités du Canada anglais, ils adhèrent volontiers au conformisme politique du rapprochement entre Français et Anglais en vigueur dans les institutions nationales ${ }^{54}$. Ce petit groupe trouvera dans les «bons-ententistes» de Québec, Camille Roy et Arthur Maheux, des collaborateurs précieux, quoique moins réguliers, pour faire contrepoids aux nationalistes de Montréal.

Il convient de noter, parallèlement à l'activité de ses sociétaires, le rôle moteur de la Société royale dans le développement des études historiques au Canada par l'institution d'un prix annuel en 1926 - la médaille Tyrrell qui souligne la contribution exceptionnelle d'un auteur à l'historiographie canadienne - et l'administration de programmes de bourses en collaboration avec la Fondation Carnegie

53. Gustave Lanctot, directeur de la section française aux Archives nationales de 1919 à 1937, puis archiviste du Dominion de 1937 à 1948, a été président de la SRC en 1948-1949. Hormis Marius Barbeau, dont 22 articles ont été publiés dans les Mémoires de la Société entre 1914 et 1946, Lanctot est le plus prolifique avec 13 publications entre 1926 et 1952 . Voir Pierre Savard, «Gustave Lanctot et la Société royale du Canada», 249. Séraphin Marion, archiviste aux Archives nationales de 1925 à 1955, a été responsable des publications en langue française à la Société de 1940 à 1952, secrétaire correspondant en langue française (1940) puis secrétaire exécutif de la SRC de 1945 à 1952. Francis-Joseph Audet, archiviste aux Archives nationales, a été président de la section française en 1926-1927. Enfin, Rodolphe Lemieux a été député et ministre fédéral sous Laurier, président de la Chambre des communes de 1921 à 1930 avant d'être nommé sénateur la même année. Parfaitement bilingue, il a souvent été présenté comme le promoteur le plus crédible de la bonne entente entre Canadiens français et Canadiens anglais. Il s'est également distingué comme professeur d'histoire du droit à l'Université de Montréal, par ses travaux historiques à la SRC et par une série de conférences à la Sorbonne en 1928 sur l'évolution politique du Canada, conférences qui avaient un temps été promises à Lionel Groulx. Comble d'ironie, Léo-Paul Desrosiers, qui travaille alors comme traducteur à la Chambre des communes, aurait servi de «nègre» pour Lemieux et rédigé les conférences en s'inspirant des travaux du très nationaliste chanoine. C'est du moins la version que laisse entendre Lionel Groulx. Voir Mes mémoires, 2: 1920-1928 (Montréal, Fides, 1971), 168-169.

54. Séraphin Marion échappe peut-être à cette tendance politique. En tout cas, Marcel Trudel, lors de son arrivée au département d'histoire de l'Université d'Ottawa, s'est étonné de le trouver occupé «à pourfendre les Anglais avec la ferveur d'une Jeanne d'Arc» dans ses cours d'histoire du Canada. Marcel Trudel, op. cit., 257. 
(1931-1943), puis avec le gouvernement du Québec ${ }^{55}$. Mais l'initiative la plus importante consiste en la fondation en 1907 de la Historical Landmarks Association lors d'une réunion de la Section $\mathrm{II}^{56}$. Elle est alors constituée en vue de la célébration du tricentenaire de la fondation de Québec et dans le dessein plus général d'identifier et de préserver les sites historiques à travers le Canada. Sans être affiliée formellement à la Société royale, l'association (qui en 1919 a changé de nom pour celui de Historic Sites and Monuments Board) se réunit en même temps et présente parfois ses comptes rendus à la Section II. En mai 1922, lors de sa réunion annuelle, la Historic Sites and Monument Board of Canada subit une transformation majeure et devient la Canadian Historical Association. Un comité avait été mis en place l'année précédente, afin d'en élargir les cadres et le mandat et de préparer une nouvelle constitution. La Canadian Historical Association continue pendant une trentaine d'années de tenir ses réunions annuelles au même endroit et en même temps que la Société royale (excepté en 1927, 1931, 1934, 1935), tout en entretenant, durant un certain temps, des rapports plus ou moins étroits avec la Section II qui l'avait enfantée. Cela explique en partie pourquoi la Canadian Historical Association, qui se veut nationale par ses objectifs et son membership, sera perçue à ses débuts comme un bastion anglophone ${ }^{57}$. Seul le petit groupe «outaouais» déjà actif à la Société royale participera de façon significative à ses travaux ${ }^{58}$.

55. Un comité de bourses est institué en 1943 à la Section I afin de distribuer une bourse annuelle de $1200 \$$ - payée par le secrétariat de la province de Québec - à un étudiant qui est déjà détenteur d'un diplôme universitaire et qui désire parfaire sa formation dans des universités européennes ou américaines. Jean Bruchési n'est sans doute pas étranger à l'initiative: admis à la Section I en 1940, il participe directement à l'attribution des bourses à titre de sous-secrétaire de la province de Québec. Voir "Rapport de la Section I», Mémoires et comptes rendus de la Société royale du Canada, $3^{e}$ série, 39 (1945): 50.

56. R. A. Preston, «The Canadian Historical Association», dans The Canadian Historical Association. Index to the Annual Report 1922-1951 (Ottawa, The Canadian Historical Association, 1952), v-vi; Carl Berger, op. cit., 31-32.

57. Pierre Savard, en se basant sur Aegidius Fauteux, rapporte un incident lors de la première réunion de la Canadian Historical Association qui est resté dans les mémoires: le juge Prud'homme, «le seul Canadien français à présenter une communication devait prendre la parole après deux ou trois communications en anglais: il avait à peine ouvert la bouche qu'un membre de langue anglaise proposa que le travail en français fut considéré comme lu»: "Gustave Lanctot et la Société royale du Canada», Les Cahiers des dix, 48 (1993): 243-244.

58. Les seuls présidents canadiens-français de la Canadian Historical Association avant 1950 appartiennent à ce groupe: Thomas Chapais en 1925-1926, Rodolphe Lemieux en 19291930, Francis-Joseph Audet en 1934-1935, Gustave Lanctot en 1940-1941 et Arthur Maheux en 1948-1949. Quant aux réunions annuelles de la CHA, les seuls qui aient présenté plus d'une communication sont: Lanctot (11), Audet (5), Maheux (3), Gérin (2) et Aristide BeaugrandChampagne (2), seul Montréalais du groupe. Voir The Canadian Historical Association. Index to the Annual Report 1922-1951 (Ottawa, The Canadian Historical Association, 1952). 
Pour la majorité des membres, ceux dont la présence est rare, la Société royale constitue avant tout une institution honorifique qui consacre la réussite littéraire et scientifique. Même Lionel Groulx, pourtant assez froid face à l'institution, ne dédaignera pas l'honneur d'en faire partie, quitte à côtoyer des adversaires idéologiques, tels Thomas Chapais, Benjamin Sulte et Pascal Poirier qui s'étaient opposés à son admission ${ }^{59}$. Certains ont vu dans le processus de cooptation des membres de la Société, où seraient mises de côté les questions d'allégeance politique, le triomphe du principe de compétence ${ }^{60}$. Or, d'autres considérations que la seule contribution aux lettres canadiennes semblent entrer en jeu. Des questions d'humeur, d'incompatibilité de caractère, des critères régionaux - choisir un Acadien ou un $\mathrm{Ca}$ nadien français de l'Ouest - des rivalités entre Montréal, Québec et Ottawa favorisent parfois l'élection de tel candidat aux dépens de tel autre mieux qualifié. L'épisode de la candidature du turbulent JeanCharles Harvey illustre l'existence d'un certain conformisme social parmi les membres, qui transcende l'opposition entre nationalistes et bons-ententistes, et qui tend à faire de l'acceptabilité sociale d'un candidat, un critère d'admission ${ }^{61}$. C'est à la suite de la candidature malheureuse de Harvey que les membres de la Section I adoptent à l'unanimité une résolution stipulant que «désormais les candidats devront s'abstenir de solliciter des votes ${ }^{62}{ }^{2}$. Le principe de la reconnaissance entre les pairs, qui s'active à l'interne à des fins de consécration «littéraire», continue donc d'être subordonné à des critères externes, signe d'un degré d'autonomie encore assez faible.

Les intellectuels canadiens-français sont toutefois jalousement attachés à l'autonomie de la Section I à l'intérieur de la Société royale. Carl Berger va même jusqu'à parler d'une tendance parmi ceux-ci à vouloir identifier la Section I au groupe ethnique des Canadiens français $^{63}$. Ainsi, Marius Barbeau propose en 1918 que tous les travaux

59. Lionel Groulx, Mes mémoires, 1: 1878-1920, 312-313.

60. Voir Pierre Rajotte, loc. cit., 389.

61. Jean-Charles Harvey mordra la poussière à deux reprises en 1935 et 1936. Son livre Les demi-civilisés, qui s'attaquait violemment à la pauvreté intellectuelle de l'élite canadiennefrançaise et à sa soumission à la puissance cléricale, avait été mis à l'index dès sa sortie en 1934 par le cardinal Villeneuve. Harvey fut également congédié de son poste de rédacteur en chef du Soleil. Lionel Groulx raconte dans ses Mémoires, les démêlés qu'il a eus avec Harvey, à la suite de son refus d'appuyer sa candidature après que celui-ci l'eut sollicité. Voir Mes mémoires, 3: 1926-1939 (Montréal, Fides, 1971), 348-353; Pierre Savard relate également l'épisode. Selon ce dernier, il aurait été dans les mœurs de la Société de multiplier les démarches - visites à domicile, lettres de sollicitation, pressions exercées par les alliés du candidat - afin de s'assurer les appuis nécessaires à l'élection. Pierre Savard, «Gustave Lanctot...», 241 et ss.

62. «Rapport de la Section I», Mémoires et comptes rendus de la Société royale du Canada, $3^{\mathrm{c}}$ série, 32 (1938): 51.

63. Voir Carl Berger, op. cit., 89. 
produits en français, même ceux des sections scientifiques, soient incorporés aux mémoires publiés de la Section $1^{64}$. Deux ans plus tard, on alla encore plus loin en discutant le projet d'incorporer les sciences de langue française aux sujets embrassés par la Section I, mais sans arriver à une conclusion unanime ${ }^{65}$. L'épisode autour de l'élection de Marie-Victorin à la Section I en 1924 illustre également cette tendance. C'est que la candidature de ce dernier à la Section V (biologie) n'a pas recueilli la majorité des voix l'année précédente. Étant donné que cette section ne compte aucun membre francophone, les sociétaires de la Section I y ont vu un effet de préjugé à l'endroit des Canadiens français et ripostent en accueillant Marie-Victorin dans leurs rangs l'année suivante ${ }^{66}$. Par ailleurs, la proposition faite en 1940 d'amalgamer les Sections française (I) et anglaise (II), en une seule section consacrée à la littérature, à la philosophie et à l'histoire canadiennes, afin de libérer une section qui serait consacrée aux sciences sociales, fut rejetée à l'unanimité des membres présents ${ }^{67}$. Lorsque s'affrontent deux appartenances, la filiation ethnolinguistique et l'affiliation disciplinaire, les intellectuels canadiens-français choisissent encore la première.

Les activités de la Section I dans le domaine de l'histoire, après avoir été très intenses durant les années 1920 et 1930, sont à la baisse durant les années 1940. Moins active comme lieu de production, la Section I perd également le monopole qu'elle exerçait comme espace de consécration. C'est qu'entre-temps, d'autres lieux d'élaboration, spécifiques à l'histoire, ont vu le jour: la Société canadienne d'histoire de l'Église catholique, la Société des dix, l'Institut d'histoire de l'Amérique française, ainsi qu'une vingtaine de sociétés historiques locales.

\section{La Société canadienne d'histoire de l'Église catholique}

À l'automne de 1932, l'American Catholic Historical Association tient pour la première fois sa réunion annuelle en sol canadien, à Toronto, aux côtés de l'American Historical Association. C'est alors que James Kenney, fonctionnaire aux Archives nationales d'Ottawa, prend l'initiative de former un comité ayant pour but de jeter les bases

64. Ibid.

65. «Procès-verbal de la Section I», Mémoires et comptes rendus de la Société royale du Canada, $3^{\mathrm{e}}$ série, 14 (1920): xviii-xix.

66. Pour un récit complet de l'épisode, voir Luc Chartrand, Raymond Duchesne et Yves Gingras, op. cit., 311-312.

67. «Rapport de la Section I», Mémoires et comptes rendus de la Société royale du Canada, $3^{\text {e }}$ série, 24 (1940): 53. 
d'un groupement analogue au Canada, projet qui se concrétise en mars 1933 lors d'une réunion au Jesuit Seminary of Philosophy à Toronto ${ }^{68}$. L'abbé Ivanhoë Caron, sous-archiviste de la province de Québec, qui est en contact fréquent avec Kenney, joue un rôle instrumental dans l'ouverture de l'institution à l'élément français. La Société, placée sous la présidence honorifique du cardinal Villeneuve, est organisée l'année suivante en deux sections autonomes, l'une anglaise, l'autre française. La section française réunit surtout des clercs-historiens, mais aussi quelques laïques reconnus comme bons catholiques ${ }^{69}$.

Les congrès annuels, tenus alternativement dans une ville de langue française et dans une ville de langue anglaise, sont des lieux de production et de recherche féconds. Plus d'une dizaine de communications sont présentées annuellement, les plus importantes étant ensuite publiées dans le Rapport annuel de la Société, ouvrage de quelque 300 à 400 pages. On y inaugure également la formule du colloque, une première à notre connaissance: ainsi à Sherbrooke en 1940, les travaux portent sur les Problèmes religieux dans le Canada au lendemain de la Conquête et, en 1942 à Saint-Hyacinthe, sur La renaissance religieuse du Canada français, de 1840 à $1855^{70}$. En revanche, ces rencontres annuelles sont entourées de tout un cérémonial religieux, les séances s'amorçant chaque matin par une messe basse. De plus, on prend soin de les convoquer en milieu de semaine afin de libérer le dimanche.

Cette double fonction, de connaissance et de foi catholique, ne sera d'ailleurs pas sans créer quelques tensions. Gustave Lanctot, l'un des éléments laïques du conseil exécutif, se fait rabrouer par le père franciscain Archange Godbout qui lui reproche de juger l'attention que portait le roi de France à l'Église canadienne aux XVII ${ }^{\mathrm{e}}$ et XVIII ${ }^{\mathrm{e}}$ siècles, «non comme l'eût fait un Fénelon ou un Vincent de Paul, mais à la façon d'un philosophe saturé du libéralisme du $\mathrm{XX}^{\mathrm{e}}$ siècle ${ }^{71}$ ». En clair, cela signifie que l'étude du passé de l'Église catholique doit

68. James F. Kenney, «Secretary's Report», Rapport annuel 1933-1934. La Société canadienne d'histoire de l'Église catholique, 1 (1934): 6-7. Voir également Arthur Maheux, «Douze ans de travaux historiques», Le Canada français, 33,1 (septembre 1945): 125-128. Mis à part le Rapport annuel, les périodiques Culture (1940-) et La Revue dominicaine rendent compte des réunions annuelles.

69. Parmi les clercs, mentionnons: O. Maurault, I. Caron, L. Groulx, P. Potvin, A. Gauthier, G. Robitaille, T.- M. Charland, V. Tremblay, A. Maheux, J.-B.-O Archambault, E. Thivierge, M. Couture, L. Pouliot, etc. Parmi les laïques: P.-G. Roy, A. Fauteux, S. Marion, V. Morin, G. Lanctot, R. Douville, J. Bruchési. Rapports annuels. La Société canadienne d'histoire de l'Église catholique (1933+).

70. Rapport 1940-1941; Rapport 1942-1943. La Société canadienne d'histoire de l'Église catholique.

71. Culture, 4,1 (mars 1943): 101. 
aussi répondre à des fins apologétiques. À cette difficulté de concilier les lumières de la foi et celles de la science s'ajoute l'ambiguité liée aux objectifs poursuivis par la Société, c'est-à-dire, «d'encourager les travaux historiques et de stimuler l'intérêt du public pour l'histoire de l'Église ${ }^{72} \gg$. Cette double nature de l'association, comme lieu d'échange pour les chercheurs et comme lieu de diffusion pour le public, est attestée par la production historiographique où se côtoient les travaux de vulgarisation et les études dotées de tout l'apparat scientifique indispensable. Or les congrès semblent demeurer largement «ignorés du public ${ }^{73}$ » pour se limiter aux discussions entre spécialistes.

\section{La Société des dix}

En 1935 la Société des dix voit le jour à Montréal. Ni «groupement professionnel ni société d'études historiques», cette association est conçue, dans l'esprit de ses fondateurs, comme «une collaboration scientifique et littéraire entre dix écrivains désireux de s'entraider dans leurs recherches historiques ${ }^{74}{ }^{\Downarrow}$. À cette fin, les sociétaires se réunissent à dix reprises durant l'année. Parmi les membres fondateurs, on compte six archivistes ou bibliothécaires (Gérard Malchelosse, Aegidius Fauteux, E.-Z. Massicotte, F.-J. Audet, P.-G. Roy et Montarville Boucher de la Bruère), deux clercs (Olivier Maurault et Albert Tessier) et deux membres des professions libérales (le notaire Victor Morin et l'architecte Aristide Beaugrand-Champagne). Le recrutement des membres s'effectue par cooptation, en fonction de leur «valeur d'historien», mais aussi de leur «esprit de confraternité t5 $^{75}$. Chacun des membres de cette fraternité littéraire s'engage à fournir son article annuel. Ces travaux sont réunis dans Les Cahiers des dix dont la publication commence l'année suivante. Réunie par un «même culte pour le passé canadien ${ }^{76} »$, cette aristocratie de l'esprit est persuadée de sa mission: diffuser la littérature historique et contribuer en cela à l'instruction des Canadiens français. Ce qui n'empêche pas, parallèlement aux travaux proprement savants, de faire la part belle aux délassements. Outre la production et la diffusion des travaux, les

72. «Statuts», Rapport annuel 1933-1934. La Société canadienne d'histoire de l'Église catholique, 1 (1934): 92.

73. Thomas-Marie Charland, «La Société canadienne d'histoire de l'Église», La Revue dominicaine, 48 (1942): 245.

74. Victor Morin, «Les dix», Les Cahiers des dix, 1 (1936): 26; Raymond Douville, «La Société des dix après un demi-siècle. Son histoire - ses membres - son œuvre», Les Cahiers des dix, 45 (1990): 217-256.

75. Raymond Douville, loc. cit., 225.

76. Aegidius Fauteux, «Préface», Les Cahiers des dix, 1 (1936): 5. 
activités des dix consistent en «des rosseries, des repas gastronomiques et des pèlerinages historiques ${ }^{77}$ ».

\section{Les sociétés d'histoire locale et régionale}

Le Canada français connaît à partir de 1925 une véritable vague de création de sociétés d'histoire locale et régionale. Jusqu'alors, on n'y en dénombrait que trois: la Société historique de Montréal (1857), la Société d'archéologie et de numismatique de Montréal (1862) et la Société historique de Saint-Boniface au Manitoba (1902). Durant les deux décennies suivantes, une vingtaine de ces associations s'ajoutent, regroupant plus d'un millier de membres ${ }^{78}$.

Les objectifs poursuivis sont, à peu de choses près, les mêmes d'une société à l'autre: étudier l'histoire locale et régionale, ce qui signifie aussi, la faire connaître et aimer; recueillir, conserver et mettre en valeur les documents qui s'y rapportent; protéger et promouvoir le patrimoine historique local et régional. Cette défense et illustration du passé s'accompagne parfois d'ambitions plus vastes: la Société historique de Québec invite à «tirer des leçons de notre histoire un enseignement pour l'avenir» et celle du Saguenay, à «dégager de cette histoire [régionale] tout ce qui peut attacher aux saines traditions, nourrir la fierté et le patriotisme, éclairer et préparer l'avenir ${ }^{79}$ ».

Les réunions sont généralement mensuelles ${ }^{80}$, la pièce de résistance constituant une conférence suivie d'une discussion entre les

77. Victor Morin, loc. cit., 26-27.

78. La Société historique du Saguenay (1924); la Société trifluvienne d'histoire régionale (1926); La Société d'histoire des Cantons de l'Est (1927); la Société historique et littéraire acadienne (1928); la Société historique de Joliette (1929); la Société historique de Rigaud (1931); la Société historique d'Ottawa (1933); la Société nicolétaine d'histoire régionale (1935); la Société historique de Sorel (1936); la Société d'histoire régionale de Rimouski (1936); la Société d'histoire régionale de Québec (1937); la Société d'histoire régionale de Saint-Hyacinthe (1937); la Société régionale du Nord de l'Outaouais (1939); la Société d'histoire du Cap-de-laMadeleine (1939); la Société historique de Sainte-Thérèse-de-Blainville (1939); la Société historique Saint-Joseph d'Alma (1942); la Société historique du nouvel Ontario (1942); la Société historique de Valleyfield; la Société historique de la Beauce. Pour un aperçu des sociétés d'histoire durant les années 1930 et 1940, voir Archange Godbout, "Les sociétés historiques de langue française», Culture, 3,1 (mars 1942): 67-89; Bulletin des sociétés historiques, 1942 (1943), $175 \mathrm{p}$.

79. Archange Godbout, op. cit., 80, 82.

80. La fréquence des réunions varie beaucoup d'une société à l'autre: la Société historique et littéraire acadienne, en raison de la dispersion de ses membres qui habitent la Louisiane et les quatre provinces maritimes, ne tient qu'une réunion annuelle, alors que les membres de la Société historique du Saguenay se réunissent toutes les deux semaines, excepté en été. D'une année à l'autre, le rythme des réunions diffère grandement. À la Société historique de Joliette, entre 1929 et 1951, de une à onze séances annuelles ont été tenues: Louis-Guy Gauthier, Société historique de Joliette: procès-verbaux, index: 1929-1982 (Joliette, Édité par Réjean Olivier, 1987), ix. 
membres. Les sujets ne diffèrent guère de ceux présentés à la Société royale - biographies, monographies locales, anecdotes historiques si ce n'est que le localisme et le pointillisme y sont encore plus poussés $^{81}$. Outre ces réunions fermées, destinées aux seuls membres, les activités sont orientées vers la diffusion publique des connaissances. Les périodiques sont toutefois rares et leur vie est éphémère: les sociétés historiques de Montréal et de Saint-Boniface (1911-1916) ont renoncé après quelques années, faute de financement adéquat ${ }^{82}$. Seul le Bulletin de la Société historique du Saguenay, organe de liaison interne fondé en 1946 et devenu en 1954 la revue Saguenayensia, a une existence durable. La publication d'ouvrages est plus fréquente: des initiatives du genre sont patronnées par les sociétés historiques de Montréal, du Saguenay, de Saint-Hyacinthe et de Trois-Rivières, cette dernière, animée par l'abbé Albert Tessier, ayant dès 1940, «une quarantaine d'ouvrages à son actif représentant quelque 40000 exemplaires $^{83}$ ». Notons l'effort de publication de sources et de documents d'archives, notamment par la Société historique de Montréal avant la refonte de ses statuts en $1916^{84}$, et par la Société historique du nouvel Ontario, avec la collection Documents historiques publiées depuis 1942. Mais c'est surtout dans la presse locale que ces associations trouvent un débouché naturel et un véhicule pour rendre compte de leurs travaux.

Les autres activités relèvent de ce que Jean-Pierre Chaline a appelé «l'incitation culturelle ${ }^{85}$ », ces sociétés se fixant pour but de faire partager le savoir qu'elles détiennent parmi les milieux cultivés et d'y faire accéder, par l'instruction, les couches populaires. L'incitation est multiforme, allant du simple encouragement jusqu'à la création d'équipements culturels. Des dépôts d'archives relatives à l'histoire régionale et locale sont créés dans la plupart des sociétés d'histoire. La très dynamique Société d'histoire du Saguenay met sur pied une bi-

81. Les travaux lus à la Société historique de Rigaud en 1942 sont caractéristiques de ce type de production. Mentionnons, entre autres, une biographie des quinze curés de la paroisse des Cèdres depuis 1767 par l'abbé Élie-J. Auclair, «Les débuts du Collège Bourget» par le père Gaspard Ducharme, «Le docteur Luc-Hyacinthe Masson, chef patriote du Nord et premier député de Soulanges» par Robert-Lionel Séguin et «Sainte-Marthe et ses Irlandais» par le père John Downs. «Rapport de la Société historique de Rigaud, 1942», Bulletin des sociétés historiques 1942 (1943): 43-45.

82. Archange Godbout, op. cit., 73.

83. Ibid., 75 .

84. Fondée en 1857 par Jacques Viger dans le but de recueillir et de publier des documents relatifs à l'histoire du Canada, la Société historique de Montréal modifie, à l'instigation de son président Victor Morin, ses statuts et règlements et ajoute parmi ses fins, celles «d'acquérir et de répandre la connaissance de l'histoire canadienne [...] par la recherche et la publication»: Archange Godbout, op. cit., 69.

85. Jean-Pierre Chaline, op. cit., 170. 
bliothèque, un département photographique et un musée ouverts au public, organise en 194115 conférences publiques et 31 projections du film du centenaire du Saguenay (1938), participe à la restauration du Site du Poste de Métabetchouan et à la mise en valeur de la chapelle de Tadoussac ${ }^{86}$. Certains bâtiments historiques sont acquis par ces groupements, tels le Château Ramezay à Montréal et le Manoir Boucher de Niverville à Trois-Rivières où la Société d'archéologie et de numismatique de Montréal et la Société trifluvienne d'histoire régionale y soutiennent respectivement un musée d'archéologie canadienne et un musée de l'industrie des arts domestiques. La Société historique de Montréal participe également à l'érection de monuments et plaques commémoratives et fait pression sur les commissions des monuments historiques du Québec et du Canada pour l'ajout de plusieurs autres ${ }^{87}$.

Qui sont les principaux animateurs de ces sociétés savantes, ceux qui les créent et en assurent la notoriété? Le portrait global pourrait se ramener à quelques traits: des hommes d'âge mûr, de milieux relativement aisés ${ }^{88}$. Les cadres de la société traditionnelle, clercs et professions libérales, dominent en province, alors que la présence des employés de l'État est marquée dans les villes d'Ottawa, Québec et Montréal. Dans les sociétés d'histoire locale, les membres des professions libérales monopolisent souvent les postes honorifiques, mais la figure la plus fréquemment rencontrée est celle du clerc enseignant dans quelque collège de province ${ }^{89}$. Quant aux sociétés savantes «nationales» (Société royale du Canada, Société canadienne d'histoire de l'Église catholique, Société des dix), l'examen attentif de leur membership durant les années 1930 et 1940 révèle la présence mar-

86. Archange Godbout, op. cit., 81 .

87. Parmi les réalisations de la Société historique de Montréal, mentionnons l'obélisque de la Place d'Youville, l'érection en 1930 du monument de Louis-Hippolyte LaFontaine en face de la bibliothèque municipale et l'apposition, en 1933, de la plaque en l'honneur de Jacques Viger, à l'entrée ouest de l'hôtel de ville. Voir Léo Beaudoin, La Société historique de Montréal: 135 ans déjà (Montréal, La Société historique de Montréal, 1994), 22.

88. Nous n'avons recensé que deux seules femmes, les deux appartenant à la Société historique de Montréal, Marie-Claire Daveluy et Albertine Ferland-Angers, cette dernière étant la première femme à être élue au Conseil de la Société historique de Montréal (1944), en plus d'assumer, trois ans plus tard, la présidence du congrès de la Société canadienne d'histoire de l'Église catholique. Réginald Hamel, John Hare et Paul Wyczynski, Dictionnaire des auteurs de langue française en Amérique du Nord (Montréal, Fides, 1989), 507.

89. Appartiennent à ce groupe des personnalités telles Victor Tremblay (Chicoutimi), Alphonse Fortin (Rimouski), Michel Couture (Sherbrooke), J.-B.-O. Archambault (Saint-Hyacinthe), Paul-Émile Farley (Joliette), Alphonse Gauthier (Rigaud), Antoine d'Eschambault (Saint-Boniface) et Lorenzo Cadieux (Sudbury). 
quée de fonctionnaires œuvrant dans le domaine culturel $^{90}$ : huit archivistes, six bibliothécaires et un traducteur, soit $40 \%$ des effectifs historiens $(15 / 40)$. Les clercs demeurent toutefois les plus nombreux avec 19 membres sur 40, soit près de la moitié.

L'univers associatif canadien-français révèle également la présence d'un participant du «troisième type», qui se distingue moins par ses caractéristiques socioprofessionnelles que par sa propension à multiplier les adhésions et à collectionner les présidences. Dans la participation à ces sociétés savantes, il est parfois difficile de distinguer les motivations hautement proclamées - le goût pour les recherches, la curiosité intellectuelle, la défense du patrimoine - des raisons moins avouables: la recherche des signes de reconnaissance sociale ou le désir de rehausser sa carte de visite. Chez certains, tel Gustave Lanctot, la poursuite des honneurs semble avoir constitué un facteur déterminant, comme l'a montré Pierre Savard ${ }^{91}$. L'analyse en termes d'ambitions personnelles est toutefois réductrice et ne rend pas justice à ces hommes des sociétés savantes dont la contribution à la promotion de l'histoire et à l'avancement du savoir a souvent été essentielle.

Le cas sans doute le plus étonnant, par la diversité de ses activités et de ses interventions, notamment dans le domaine de l'histoire, est celui du notaire Victor Morin (1865-1960) ${ }^{92}$. Ce dernier amorce en 1890 une longue carrière de juriste - 70 ans! - remplie d'honneurs: trésorier (1897-1930) puis président (1930-1933) de la Chambre des notaires, notaire titulaire de la Corporation de la ville de Montréal de 1897 à 1910, professeur de droit administratif à la Faculté de droit de l'Université Laval de Montréal de 1909 à 1919, puis professeur de procédure notariale de 1919 à 1939, membre en 1930 de la Commission d'étude du code civil en ce qui a trait aux droits de la femme, sans compter ses nombreuses fonctions à la tête de compagnies d'assurances. Victor Morin a été membre de plus d'une douzaine d'associations et a accumulé les postes honorifiques: membre-fondateur de la Société des dix (1935-1959), membre de la Commission des monu-

90. Nous avons dénombré systématiquement tous les membres consacrés comme historiens à la Société des dix et à la Société d'histoire de l'Église catholique entre 1930 et 1950, ainsi que les membres de la Section I de la Société royale dont la production écrite les qualifiait comme historiens.

91. Pierre Savard, «Gustave Lanctot et la Société royale du Canada», loc. cit., 238-250.

92. Voir « $\mathrm{M}^{\mathrm{e}}$ Victor Morin: $6^{\mathrm{e}}$ président (1916-1928)», dans Phillippe La Ferrière, Centenaire de la Société historique de Montréal (1858-1958) (s.l., s.é., 1962), 46-55; Jean-Jacques Lefebvre, «Victor Morin 1865-1960», Mémoires de la Société royale du Canada (1961) $3^{\mathrm{e}}$ série, 131-138; Lionel Groulx, "Chronique de l'Institut», Revue d'histoire de l'Amérique française, 14,3 (décembre 1960): 475. 
ments historiques depuis sa constitution en 1922, président général de la Société Saint-Jean-Baptiste (1916-1928), président de la Société historique de Montréal (1916-1928), de la Société d'archéologie et de numismatique de Montréal (1940), de la Société fiduciaire du Château de Ramezay (1927-1957), de la Société royale du Canada (1940), de la Société canadienne d'histoire de l'Église catholique (1941-1942), pour ne nommer que les plus importants. Son action dans le domaine culturel et historique est loin d'être négligeable. C'est au cours de son mandat comme échevin à la Ville de Montréal, entre 1910 et 1913, qu'il propose et fait accepter par le Conseil l'établissement d'une bibliothèque municipale sur la rue Sherbrooke et qu'il fait également adopter un système de classification des archives de la ville. C'est également sous sa présidence que la Société historique de Montréal crée un prix destiné à stimuler la recherche historique, la médaille de la Société historique de Montréal (1922). À titre de président général de la Société Saint-Jean-Baptiste, il est l'un des instigateurs du grand défilé du 24 juin, rue Sherbrooke à Montréal, de l'érection au Pied-duCourant d'un monument à la mémoire des patriotes exécutés en 18381839 et de l'immense croix illuminée au sommet du mont Royal (1924). Il s'est également distingué comme organisateur de la célébration du troisième centenaire de Montréal en 1942. Féru d'héraldique, Morin a signé un ouvrage sur La science du blason et de nombreuses études sur l'histoire de Montréal. Homme d'appareil et de loi, il est l'auteur du fameux code de procédures délibérantes qui porte son nom, en plus de se faire l'historiographe de la Société royale du Canada et de la Société historique de Montréa ${ }^{93}$. On se surprend qu'il ait eu le temps, à travers tout cela, de se marier deux fois et d'élever 13 enfants.

L'exemple de Morin démontre que l'on aurait tort de limiter la perception des fonctions de ces sociétés savantes à la consécration d'une classe de «notables culturels». Certes, l'essor des sociétés savantes est à mettre en relation avec l'épanouissement d'une sociabilité urbaine, dont elles constituent l'une des formes essentielles depuis le milieu du XIX ${ }^{e}$ siècle. L'enracinement, entre autres, des sociétés d'histoire locale dans les centres de province, entre 1920 et 1950 , satisfait à la fois la curiosité légitime pour le passé régional et les ambitions d'une élite locale qui profite des postes honorifiques et des relations que procure la participation à ces associations culturelles,

93. La science du blason; ses principes, ses règles et leur application (Montréal, Les Éditions des dix, 1958), 42 p.; «Les origines de la Société royale du Canada», Les Cahiers des dix, 2 (1937); «L'odyssée d'une société historique (Société historique de Montréal), Les Cahiers des dix, 8 (1943). 
pour ajouter à son crédit politique un capital symbolique et un capital social indispensables pour prétendre au leadership naturel et moral des Canadiens français. Mais les activités savantes occupent au sein de ces associations la plus grande part. De plus, ce sont ces sociétés qui amorcent le processus de reconnaissance sociale de l'historien, comme producteur distinct dans le champ des lettres canadiennes, tout en participant à la promotion de l'écriture de l'histoire durant les années 1930 et 1940. Il est difficile d'établir une mesure du progrès qualitatif et quantitatif de l'historiographie canadienne-française durant cette période. Là n'est d'ailleurs pas notre propos. La consultation rapide de quelques inventaires bibliographiques du début des années 1940 révèle néanmoins des chiffres impressionnants: 582 ouvrages et articles à caractère historique publiés en français au Canada sont dénombrés pour la seule année 1942 dans le Bulletin des sociétés historiques canadiennes-françaises, et 345 dans la revue Culture pour les années 1945 et 1946. L'action structurante des sociétés d'histoire n'est sans doute pas étrangère à cette production. L'image du vide pour représenter la période de l'avant-1947 dans le domaine de l'histoire au Canada français s'accorde donc mal à la réalité.

$\mathrm{Ne}$ tombons toutefois pas dans l'autre extrême. La contribution de ces sociétés savantes à la genèse de la discipline historique au Canada français n'est effective que dans la mesure où elles compensent son faible développement dans le système universitaire. Les structures d'encadrement qu'elles proposent sont trop lâches pour supporter un groupe de chercheurs à temps plein. De plus, le processus de disciplinarisation de l'histoire s'y limite à sa spécification comme genre littéraire et comme domaine d'études, l'étude du passé y demeurant soumise à des préoccupations littéraires, politiques et idéologiques. L'autonomisation de l'histoire par rapport à ces enjeux, ce sont les fondations de 1947 qui l'impulseront, en créant les conditions institutionnelles propices à un tel désengagement.

\section{LES FONDATIONS DE 1946 ET 1947: POUR UNE VISION NUANCÉE}

L'apparition dans le système universitaire de nouveaux espaces de production et de formation spécifiques à l'histoire n'est pas le résultat d'une naissance spontanée. Le contexte socio-économique de l'aprèsguerre est favorable à l'expansion des institutions de haut savoir. La démographie croissante, l'enrichissement des salariés et la tertiarisation de l'économie suscitent une forte demande en services publics. L'activité intellectuelle elle-même s'intensifie, le nombre de livres publiés au Canada français ayant quintuplé durant la guerre 
selon le sous-secrétaire de la province, Jean Bruchési ${ }^{94}$. Cette montée impressionnante doit être liée en bonne partie, il est vrai, aux problèmes que connaît alors l'édition en France. N'empêche. De plus, certains des obstacles qui entravaient plus spécifiquement le développement des études historiques dans le système universitaire sont levés. Les collèges classiques de la région de Montréal, à court de clercs, acceptent désormais d'embaucher des laïques, suivis par certains collèges de la région de Québec ${ }^{95}$. En outre, le déménagement de l'Université de Montréal sur les flancs du mont Royal en 1942 ainsi que le lancement d'une campagne de souscription financière en octobre 1947, la première depuis celle de 1919-1920, créent un environnement physique et financier favorable aux expansions.

L'idée de mettre sur pied un programme spécialisé en histoire circule depuis quelques années déjà. A l'Université de Montréal, le doyen de la Faculté des lettres, M $^{\text {gr }}$ Émile Chartier, évoque dès 1942 la création d'un institut d'histoire autonome ${ }^{96}$. À Québec, l'opportunité d'une telle fondation est l'objet de disputes depuis une décennie. Camille Roy, qui a quitté le poste de recteur pour celui de doyen de la nouvelle Faculté des lettres en 1937, voit d'un bon œil la création d'un programme d'enseignement spécialisé en histoire. Les dirigeants de la Faculté des arts, Arthur Maheux en tête, perçoivent l'ajout d'un institut d'histoire comme une façon de redorer le blason d'une faculté moribonde ${ }^{97}$. C'est finalement la Faculté des lettres qui emportera la mise, Maheux étant placé à la tête du nouvel institut d'histoire, à la condition expresse de ne pas enseigner l'histoire du Canada (afin d'éviter les remous que sa position «bon-ententiste» avait créés au sein des milieux nationalistes ${ }^{98}$ ).

Sans être une création spontanée, la fondation de l'Institut d'histoire et de géographie de l'Université Laval a été largement improvisée. Mis au courant du projet montréalais, les dirigeants de l'Université Laval en ont dessiné les grandes lignes à la vapeur avec le concours de la Société historique de Québec ${ }^{99}$. Le programme d'études de la première année en 1947-1948 — une belle «salade», selon l'ex-

94. «Rapport de la Section I», Mémoires et comptes rendus de la Société royale du Canada, $3^{\text {e }}$ série, 36 (1942): 48.

95. Marcel Trudel, op. cit., 144.

96. L'Action universitaire, 8 (septembre 1942): 29; «L'avenir de la Faculté des lettres», Annuaire 1943-1944. Université de Montréal. Faculté des lettres, 58-59.

97. Jean Hamelin, Histoire de l'Université Laval, op. cit., 169 et 183.

98. Centre de recherches Lionel-Groulx. Fonds Lionel-Groulx. Correspondance avec Maurice Lebel (Institut d'histoire et de géographie de l'Université Laval). P1/A, 2189. Lettre de M. Lebel à L. Groulx, 1er décembre 1947.

99. Voir Jean Hamelin, op. cit., 183. 
pression de Maurice Lebel - prévoit des cours d'histoire canadoaméricaine (Marcel Trudel), de folklore canadien et de linguistique canadienne (Luc Lacourcière), de géographie humaine de l'Amérique du Nord (Marius Barbeau), d'allemand (Joseph Belleau), de méthodologie et de relations politiques et intellectuelles entre l'Europe et l'Amérique (André Latreille), tout cela menant à l'obtention d'une licence ès lettres de culture ${ }^{100}$.

À Montréal, sous la direction ferme de Guy Frégault, le programme d'études est mieux structuré. Les cours pour l'année 19471948 sont divisés en trois sections - histoire générale, histoire du Canada et sciences auxiliaires - et y sont conçus pour orienter les étudiants, soit vers la culture générale (licence d'histoire), soit vers la spécialisation (maîtrise et doctorat ${ }^{101}$ ). À cette fin, une attention spéciale est portée à l'enseignement de la méthodologie ainsi qu'à la tenue de séminaires d'histoire. Par ailleurs, les statuts de l'université définissent l'Institut comme «le groupement de tous les professeurs enseignant une matière sous la direction d'un directeur chargé de coordonner les efforts et les programmes de leurs cours ${ }^{102} »$. L'Institut jouit donc d'une relative autonomie comme section affiliée à la $\mathrm{Fa}$ culté des lettres en plus de régir une aire d'enseignement consacrée uniquement à l'histoire, ce qui n'est pas le cas à Laval.

Les débuts de ces deux institutions sont toutefois très modestes. À Laval, un seul étudiant régulier est inscrit la première année (Claude Galarneau), trois la deuxième année et huit la troisième ${ }^{103}$. À Montréal, les diplômés des collèges classiques détenteurs d'un baccalauréat ès arts postulaient dès le départ un diplôme de second cycle. Le programme de premier cycle ne compte donc que très peu d'inscrits: sept étudiants en 1947, un en 1954, deux en 1955, neuf en 1957104. Aux études avancées, les effectifs sont plus importants: en 1947-1948, Frégault dirige dix étudiants à la maîtrise - qui doivent produire une thèse dont le minimum requis est fixé à seulement dix pages - et six

100. Centre de recherches Lionel-Groulx. Fonds Lionel-Groulx. Correspondance avec Maurice Lebel (Institut d'histoire et de géographie de l'Université Laval). P1/A, 2189. Lettre de M. Lebel à L. Groulx, $1^{\text {er }}$ décembre 1947, et «Horaire des cours 1947-1948. Certificats pour la licence ès lettres de culture».

101. Guy Frégault, «Sommaire des cours 1947-1948. Institut d'histoire», Annuaire 19471948. Université de Montréal. Faculté des lettres, 31-36.

102. Lucien Campeau, «Notes sur le département d'histoire de l'Université de Montréal», Bulletin du Centre de recherche en civilisation canadienne-française, 9 (décembre 1974): 5; «Organismes divers. Les instituts», Annuaire général 1947-1948, Université de Montréal, 278279.

103. Marcel Trudel, op. cit., 204.

104. Lucien Campeau, op. cit., 8. 
étudiants au doctorat ${ }^{105}$. Par ailleurs, les efforts initiaux pour développer l'institut montréalais en centre de recherche spécialisé se butent à des obstacles financiers et administratifs qui feront échouer le projet. La charge de travail des professeurs ne favorise pas davantage la recherche. L'enseignement entre 1947 et 1959 est essentiellement prodigué par quatre professeurs titulaires - Guy Frégault, Michel Brunet, Maurice Séguin et Antonin Papillon - , ce qui les contraint à multiplier les préparations de cours. En 1951-1952, ces derniers donnent 19 des 21 cours et séminaires différents ${ }^{106}$.

Mais par-delà la quantité de chercheurs et de connaissances produites initialement, la fondation de ces deux instituts universitaires contribue à la création d'une aire autonome d'enseignement et de recherche, à la promotion de l'histoire critique et à la formation des spécialistes en mesure d'assurer ce type de production historiographique. La voie est pour ainsi dire pavée, durant les décennies suivantes, pour la construction d'une discipline scientifique en milieu universitaire et pour l'autonomisation graduelle de l'histoire par rapport aux différentes injonctions externes (idéologiques, religieuses, doctrinales et littéraires) qui structurent encore, dans le milieu savant, l'étude du passé et la quête de la vérité. Les embûches à la conquête de cette autonomie seront plus importantes à l'Institut d'histoire de l'Amérique française, cette institution se voulant à la fois un foyer de recherche scientifique pour les historiens et un lieu de vulgarisation pour le grand public.

Rappelons que lorsqu'il est créé, l'Institut d'histoire de l'Amérique française cherche à donner «une plus vigoureuse poussée à l'étude de l'histoire du Canada français», mais aussi à «explorer et à mettre en lumière le fait français en Amérique ${ }^{107}{ }$. Donald Creighton n'a-t-il pas tracé, l'année précédente dans les pages de la Canadian Historical Review, un programme symétrique pour l'étude de l'histoire de l'Amérique britannique ${ }^{108}$ ? À ce dessein général s'ajoutent des objec-

105. Archives de l'Université de Montréal. Fonds du département d'histoire. Dossiers particuliers. E16/ D1, 2, 44 (Guy Frégault), «Analyse de fonctions (Guy Frégault, 1948)»: 3-5.

106. «Institut d'histoire», Annuaire 1951-1952. Université de Montréal. Faculté des lettres, 56-63. Au-delà du nombre élevé de préparations de cours, c'est l'étendue des périodes historiques et des objets couverts par les professeurs qui rend leur tâche ardue: le R. P. Papillon couvre à la fois l'histoire des civilisations antiques et l'histoire du Moyen Âge, tout en continuant à enseigner parallèlement à l'Institut d'études médiévales; Michel Brunet assure à la fois les cours en histoire des États-Unis et en histoire de l'Europe contemporaine. Quant à Guy Frégault, il doit assumer en plus de sa charge d'enseignement, les tâches administratives liées à la direction de l'Institut d'histoire.

107. Le Devoir, 26 octobre 1946, cité dans Rosario Bilodeau, «Les vingt ans de la Revue d'histoire de l'Amérique française», RHAF, 20,1 (juin 1967): 2.

108. "Canada in the English-Speaking World», CHR, 26,2 (juin 1945): 119-127. 
tifs plus spécifiques visant à résoudre certains des problèmes de l'institution historienne canadienne-française. L'un de ces problèmes est l'isolement des historiens francophones, l'Institut se proposant de rassembler, à l'instar de la Canadian Historical Association, «tous les bons ouvriers de la recherche historique» et de «constituer dans toutes les provinces du fait français, des équipes de chercheurs organisés soit à l'état de sections soit à titre de membres-correspondants ${ }^{109}$ ". Le projet de fédération des sociétés d'histoire locale avait déjà été tenté en 1942 à l'occasion du lancement du seul numéro du Bulletin des sociétés historiques canadiennes-françaises. C'est ce projet que l'Institut reprend à son compte en proposant une affiliation aux sociétés d'histoire, celles-ci devant faire accepter leurs règlements par l'Institut et produire le rapport de leurs travaux lors de l'assemblée générale annuelle. L'Institut veut ainsi s'assurer que les sections locales fassent l'histoire de leur région, «selon toutes les exigences de la méthode historique», en vue d'établir une histoire qui marque les points de contact avec l'histoire plus large de l'Amérique française et dont la problématique générale serait définie lors de la réunion annuelle ${ }^{110}$. Le terme d'affiliation masque en fait une structure centralisée qui ne donne à peu près aucune visibilité aux travaux des sociétés d'histoire, tout en retirant le maximum de prestige de leur regroupement ${ }^{111}$.

Un autre des objectifs visés par Groulx est de faire de l'Institut «une grande école d'historien», hors des murs de l'université ${ }^{112}$. À cette fin, des articles méthodologiques à caractère prescriptif sont publiés dans les premiers numéros de la Revue d'histoire de l'Amérique française à l'intention des apprentis historiens. Des cours publics sont également organisés sur certaines des figures héroïques de la Nouvelle-France, de manière à attirer le plus large public possible ${ }^{113}$. Cependant, ce projet de Groulx, qui faisait double emploi avec celui des instituts universitaires, sera rapidement abandonné.

109. Roland Côté, «M. Guy Frégault dit le rôle du nouvel Institut d'histoire», Le Canada, 19 décembre 1946; Lionel Groulx, «Pages liminaires», RHAF, 1,1 (juin 1947): 4; Lionel Groulx, «Vie de l'Institut», RHAF, 2,1 (juin 1948): 155.

110. Lorenzo Cadieux, «Mémoire en vue d'un meilleur rendement de nos sociétés d'histoire régionale», $R H A F, 2,1$ (juin 1948): 147-151.

111. Les sociétés d'histoire canadiennes-françaises ont été moins biens servies par la Revue d'histoire de l'Amérique française, qui ne disait à peu près mot de leurs travaux, que par la Canadian Historical Review qui publiait systématiquement durant les années 1930 et 1940 les rapports de leurs travaux annuels.

112. Lionel Groulx, «Vie de l'Institut», RHAF, 2,1 (juin 1948): 4.

113. «Louis Jolliet» par le père Jean Delanglez en 1947; «Pierre Gaultier de Varenne, sieur de La Vérendrye» par l'abbé Antoine d'Eschambault en 1948: «Pierre Rigaud de Vaudreuil» par Guy Frégault en 1951. 
La fin principale poursuivie par l'Institut demeure la promotion de la recherche historique. Les moyens d'y parvenir consistent en la constitution de fonds d'archives, l'édition de quelques ouvrages historiques et le lancement de la Revue d'histoire de l'Amérique française. Le Canada français ne dispose alors pas de revue scientifique spécialisée en histoire. Quelques articles ainsi que des synthèses historiques sont publiés occasionnellement dans des périodiques de littérature et de culture générales comme La Revue canadienne, Le Canada français ou Culture. Quant au Bulletin des recherches historiques, on y trouve des commentaires d'archives, des études généalogiques, des monographies locales, de courtes biographies ainsi que des anecdotes historiques, mais pas de notes en bas de page ni de comptes rendus critiques qui pourraient permettre aux historiens de suivre l'évolution des recherches dans leur champ et de départager le bon grain de l'ivraie. La fondation de la Revue d'histoire de l'Amérique française permet de pallier ces insuffisances en servant à la foi d'organe de liaison entre les historiens et d'organe d'officialisation des critères de l'histoire méthodique et scientifique. La Canadian Historical Review joue pour ainsi dire ce rôle de "police discursive ${ }^{114}$ » au sein de la communauté des historiens canadiens-anglais depuis sa fondation en 1920. Mais la revue, publiée en anglais seulement, est peu lue au Canada français et les historiens francophones n'y participent guère: ils ont contribué pour moins de $2 \%$ des articles entre 1920 et 1949, selon Fernand Ouellet ${ }^{115}$.

Le rôle de la nouvelle revue dans le processus de disciplinarisation de l'histoire est fondamental dans la mesure où on cherche à y fixer de façon étanche les frontières entre l'histoire et la littérature, l'activité de connaissance et l'idéologie: "pour rien au monde, affirme Groulx, la Revue n'acceptera de se muer en instrument de propagande nationaliste», mais elle s'efforcera au contraire «de faire de l'histoire objective, scientifique ${ }^{116}{ }$. Cela dit, le seul fait de constituer l'Amérique française (ou le Canada français) comme objet d'étude est une

114. La notion de «police discursive» a été utilisée par Michel Foucault, L'ordre du discours (Paris, Gallimard, 1971) pour décrire le discours d'une discipline et par Olivier Dumoulin pour désigner la reconnaissance de pratiques et de références identiques exercée par des revues professionnelles ou scientifiques. Voir «La professionnalisation de l'histoire en France (1919-1939)», dans Historiens et sociologues aujourd'hui. Journées d'études annuelles de la Société française de sociologie, Université de Lille (14-15 juin 1984) (Paris, Éditions du CNRS, 1986), 49-59.

115. Fernand Ouellet, op. cit., 74. En fait, les seuls collaborateurs canadiens-français appartiennent au réseau outaouais des «bons-ententistes». Gustave Lanctot est le seul à avoir publié plus d'un article entre 1920 et 1950 (4); quant aux comptes rendus, Lanctot en a produit 35, Arthur Maheux 6, Léon Gérin 3, Francis-Joseph Audet 2. Seul Aegidius Fauteux, avec 4, n'appartient pas à ce groupe.

116. «Vie de l'Institut», RHAF, 2,3 (décembre 1948): 468-469. 
action indissociablement intellectuelle et politique. De plus, en dépit des professions de foi, il subsistera pendant plusieurs années une certaine ambiguïté quant au message véhiculé: revendication de sérieux, d'objectivité, de scientificité, de production de connaissances nouvelles, d'une part, et promotion de l'accessibilité et du caractère grand public de la revue, d'autre part. Cette ambiguité s'exprime par la cohabitation simultanée, à l'intérieur des pages de la revue, de deux traditions historiographiques, l'une rattachée aux formes narratives plus anciennes, l'autre au courant méthodique et objectiviste. C'est ainsi que dans une même livraison de la revue en mars 1950, Léo-Paul Desrosiers peut se plaindre de la lourdeur de l'appareil scientifique dans l'ouvrage de Marcel Trudel sur Louis XVI, le Congrès américain et le Canada (1774-1789) et Guy Frégault reprocher au Bigot de Pierre-Georges Roy, «la pauvreté de sa documentation» et «l'insouciance de sa méthode historique ${ }^{117}$ ».

Ces tensions traduisent la difficulté du passage d'une forme d'histoire traditionnelle, subordonnée à des enjeux littéraires ou idéologiques, à une histoire autonome, affranchie de ces mêmes enjeux. Ces tiraillements s'expliquent également par la situation économique précaire de l'Institut et de la Revue qui tend à faire coexister deux logiques économiques diamétralement opposées. Pierre Bourdieu en a exposé le principe dans ses travaux sur le champ littéraire ${ }^{118}$ : à un pôle, l'on retrouve une logique qui privilégie la production et ses exigences spécifiques, issues d'une histoire autonome à la discipline, cette production ne «reconnaissant d'autre demande que celle qu'elle peut produire elle-même ${ }^{119}$ ». Ce pôle, c'est celui de la discipline scientifique où les agents circulent dans un marché fermé, les producteurs de savoir constituant aussi les seuls consommateurs ${ }^{120}$. À l'autre pôle, l'on retrouve la logique économique des industries littéraires qui «faisant du commerce des biens culturels un commerce comme les autres, confèrent la priorité à la diffusion, au succès immédiat et temporaire, mesuré par exemple au tirage et se contentant de s'ajuster à la demande préexistante de la clientèle ${ }^{121}{ }^{\prime}$. Cet autre pôle s'inscrit dans un marché ouvert, où les consommateurs ne sont pas eux-mêmes producteurs de savoir.

117. RHAF, 3,4 (mars 1950): 594-602; 609-613. Jean Lamarre a rendu compte, dans les détails, de la polémique. Voir «Le salut par la science», dans Le devenir de la nation québécoise (Sillery, Septentrion, 1993), 275-309.

118. Pierre Bourdieu, Les règles de l'art. Genèse et structure du champ littéraire (Paris, Seuil, 1992), 201-237.

119. Ibid., 202.

120. Voir Yves Gingras, op. cit., 42-43.

121. Pierre Bourdieu, op. cit., 202. 
En l'absence de tout financement gouvernemental avant 1964, l'évolution de l'Institut et de la Revue est marquée par la nécessité de satisfaire à la fois le milieu fermé des spécialistes et le marché plus large du public cultivé. La confusion, entretenue par les sollicitations d'appui de la part des dirigeants, est réfléchie, comme par un jeu de miroirs, par la clientèle: ainsi, tel fabricant de biscuits (la Stuart Biscuit Co. ltd.) offre-t-il en 1947, comme cadeau des fêtes, un abonnement à la Revue à cent de ses meilleurs clients ${ }^{122}$. Pour préserver le caractère scientifique de l'entreprise, les dirigeants de l'Institut ont donc tendu à multiplier les cloisons: d'abord entre les membres ordinaires et les membres donateurs, seuls les premiers ayant droit de vote lors des assemblées générales; puis par la création en 1956 d'un organe distinct de l'Institut, la Fondation Lionel-Groulx, dont la fonction est de financer l'ensemble des activités; enfin, par la disjonction, à tout le moins dans le principe, entre l'Institut et la revue elle-même, le premier continuant à être ouvert à «tous ceux qui s'intéressent à l'histoire ${ }^{123}$ ", la seconde se constituant en château fort de l'histoire scientifique, établie de façon quasi exclusive dans les milieux universitaires.

\section{CONCLUSION}

Les fondations de 1947 ont été surtout pensées en termes de rupture avec l'antérieur. Celles-ci participent pourtant d'un processus long de disciplinarisation de l'histoire qui s'affirme plus nettement à partir des années 1920 et qui est marqué par un double mouvement de spécification et d'autonomisation. La multiplication des sociétés savantes à caractère historique durant la période de l'entre-deux-guerres a joué un rôle essentiel dans la délimitation d'un espace de production et de reconnaissance propre à l'histoire. Ces associations offrent alors un milieu d'accueil et d'encadrement à ceux qui souhaitent faire la promotion de l'histoire, tout en permettant de rompre, en partie, l'isolement dans lequel étaient plongés leurs prédécesseurs. La mise sur pied de nouveaux espaces de production et de reconnaissance au lendemain de la Deuxième Guerre mondiale, notamment dans le système universitaire, ne fait que consolider et prolonger le mouvement.

La conquête d'un savoir historique autonome a toutefois été plus difficile. Dans l'enseignement supérieur, l'histoire demeure dominée par la littérature et la philosophie, les universités canadiennes-françaises, encore attachées au programme d'étude généraliste des humanités

122. Lionel Groulx, «Vie de l'Institut», $R H A F, 1,3$ (décembre 1947): 475.

123. René Durocher, «Chronique de l'Institut», RHAF, 34,3 (décembre 1980): 497. 
classiques, tardant à mettre sur pied un enseignement spécialisé. Quant aux sociétés savantes, elles s'affirment comme des structures de sociabilité qui, parallèlement aux activités proprement savantes, font la part belle aux mondanités. De plus, leur existence est trop fragile pour supporter un groupe de chercheurs à temps plein. La quête du passé y demeure déterminée en grande partie par des enjeux sociaux, littéraires, politiques et idéologiques. Ce qu'on y cherche, ce sont ou bien des munitions pour défendre le catholicisme et la nation, ou bien un moyen de promotion et de consécration sociales. La mise sur pied des deux instituts universitaires ainsi que le lancement de la Revue d'histoire de l'Amérique française ont inauguré un nouvel espace social, un lieu désengagé, dédié à la production de connaissances historiques objectives et à la reproduction des agents en mesure d'assurer cette production.

Bref, les fondations de 1947 participent simultanément de deux dynamiques. L'une de continuité, par la poursuite de la spécification de l'histoire dans le champ intellectuel et scientifique. L'autre de rupture, par la mise en place des conditions institutionnelles propres à susciter une prise de distance par rapport aux différentes demandes qui, dans la sphère publique, prétendent structurer la figuration du passé. Au carrefour, l'on trouve l'Institut d'histoire de l'Amérique française, qui s'affirme indissociablement comme lieu de production scientifique et comme lieu d'expression des attentes sociales en matière d'interprétation du passé. Alors, 1947, un tournant dans la disciplinarisation de l'histoire au Canada français? Disons plutôt une charnière entre deux expressions momentanées du processus, l'une plus ancienne, l'autre plus moderne. 